\title{
Taking a New Contour: A Novel Approach to Panel Unit Root Tests ${ }^{1}$
}

\author{
Yoosoon Chang \\ Department of Economics \\ Rice University
}

\begin{abstract}
The paper introduces a novel approach to testing for unit roots in panels. Following Chang and Park (2004), the approach takes a new contour that is drawn along the line given by the equi-squared-sum instead of the traditional one given by the equi-sample-size. As we show in the paper, the distributions of the unit root tests based on nonlinear IV $t$-ratios (which includes the Dickey-Fuller test as a special case) are asymptotically normal along the new contour. The normal asymptotics hold under both the null of a unit root and the local-to-unity alternative. Moreover, they are applicable also for the models with intercept, as long as is used the demeaning method relying only on the past information. Subsequently, we demonstrate that this startling finding may be exploited to invent tools and methodologies for the effective inferences in panel unit root models. In particular, our theory implies that the individual tests may be regarded asymptotically as normal samples if they are computed using the samples having the same sum of squares across all cross-sectional units, which may be obtained through the standard bootstrap method. Consequently, we may conveniently use various functionals of the individual tests to do valid inferences in panels.
\end{abstract}

First Draft: December 10, 2003

This version: September 25, 2004

JEL Classification: C12, C15, C32, C33.

Key words and phrases: Panel unit root test, nonlinear IV methodology, equi-squared-sum contour, bootstrap, Cauchy estimator, order statistics.

\footnotetext{
${ }^{1}$ The paper was prepared for the presentation at Panel Workshop held at Cass Business School, London, UK, on March 15, 2004. I am very grateful to the workshop organizer Giovanni Urga for the invitation, and to Joon Park, Peter Pedroni and Donggyu Sul for many helpful discussions. I have also benefitted from the comments from the seminar participants at Sungkyunkwan University, University of Tokyo, The 11th International Conference on Panel Data, 2004 Far Eastern Econometrics Society Meeting, and 2004 NBER Summer Institute. Correspondence address to: Yoosoon Chang, Department of Economics - MS 22, Rice University, 6100 Main Street, Houston, TX 77005-1892, Tel: 713-348-2796, Fax: 713-348-5278, Email: yoosoon@rice.edu.
} 


\section{Introduction}

Inference in nonstationary panels has recently drawn much attention as more panel datasets covering long time spans became available. In particular, the unit root tests in panels have been frequently used by numerous authors to investigate many important economic interrelationships among countries or regions, which may imply convergence, divergence or parity of some sets of economic variables. The growth convergence and the purchasing power parity of exchange rates are prime examples, and routinely examined by the unit root tests applied to the time series observations across the countries or regions under investigation. The unit root tests that are applicable for the panel data have been developed by many authors including Quah (1994), Levin, Lin and Chu (2002), Im, Pesaran and Shin (2003), Maddala and Wu (1999), Choi (2001a, 2001b), Chang (2002, 2004), Chang and Song (2002), Moon and Perron (2001), Phillips and Sul (2001), Bai and Ng (2004) and Pesaran (2003). See, e.g., Banerjee (1999), Phillips and Moon (2000), Baltagi and Kao (2001) and Pedroni and Urbain (2004) for surveys on the recent development of the unit root tests in panels.

All of the existing panel unit root tests combine some transformations of the individual unit root test statistics obtained across cross-sectional units. Quite naturally, under the assumption of cross-sectional independence, the appropriately transformed and standardized combinations of individual tests are well expected to be approximately normal as the number of cross sections $N$ gets large. Indeed, virtually all the panel unit root tests rely on normal $N$-limit theory, whose validity requires appropriate normalizations of individual tests, as well as asymptotic cross-sectional independence. It is therefore fair to say that a good panel test is the one which efficiently normalizes the individual tests and effectively deals with cross-sectional dependency. In this paper, we concentrate more on efficient normalization of the individual tests. The problem of cross-sectional dependency was first addressed in Chang (2002), and dealt with by using the methodology based on nonlinear instrumental variable estimation. Subsequent authors introduced common factors in their models to allow for cross-sectional dependency.

The efficient normalization of the individual tests is much more difficult than one might think. As is well known, the individual unit root tests have null distributions that are nonstandard and nonnormal. Their time $T$-asymptotics yield distributions commonly represented by various functionals of Brownian motions, and in particular, known to be asymmetric and skewed. See, e.g., Fuller (1996) for the tabulations of them. Consequently, the standardization through the mean and variance adjustment or the $p$-value transformation, which are two most frequently used methods for normalization, often works poorly even when $T$ is relatively large. Worse, the errors made in the normalizations for individual tests are accumulated as $N$ of them are combined to compute the panel unit root test. Obviously, the problem gets worse as $N$ increases. We require, however, that $N$ tend to infinity to obtain the normal $N$-asymptotics. This is a serious dilemma. It is well known that all the existing panel unit root tests suffer from rather serious size distortions when $N$ is large compared to $T$.

To overcome this difficulty, we take a totally different method in this paper: A novel approach based on a new contour to achieve efficient normalization of the individual tests. Following Chang and Park (2004), our approach takes the contour that is drawn along the 
line given by the equi-squared-sum instead of the traditional one given by the equi-samplesize. In the paper, we extend their results for the OLS based $t$-ratio to the general nonlinear IV $t$-ratios, which are based on the instruments given by the nonlinear transformations of the lagged levels of the time series to be tested. The nonlinear IV $t$-ratios were considered earlier by Chang (2002) and Phillips, Park and Chang (2004) along the conventional equisample-size contour. More precisely, we show in this paper that the limiting distributions of the general nonlinear IV $t$-ratios are standard normal on the new contour that is given by the fixed sum of squared IV's. Our results would therefore include those of Chang and Park (2004) as a special case. As in Chang and Park (2004), our analysis extends to the models with roots local-to-unity, and also to the models with intercept if the demeaning is done recursively using only the past information.

The new asymptotics we derive along the contour of the equi-squared-sum have very important and far-reaching implications for inference in nonstationary panels. For the independent panels, it would imply that the individual unit root tests behave asymptotically as if they were independent and identically distributed standard normals, if we observe their values along the contour of the equi-squared-sum. That is, if we set the sum of squares to be the same across all cross-sectional units, the resulting individual unit root tests can be regarded as standard normal samples. This rather startling result would certainly give us a great opportunity to do effective inferences in nonstationary panels. For instance, we may now use the order statistics such as the minimum or maximum to test the null hypothesis that all (some) cross-sections have unit roots against the alternative that some (all) do not. Under the equi-squared-sum scheme, the individual tests have normal $T$-asymptotics for each $N$, regardless of being small or large, or letting it be fixed or tend to infinity. We may indeed rely on standard normal distribution theory for both $T, N$ or their joint asymptotics if we simply aggregate the individual test statistics along the new contour.

For many of the practical applications, the panels are observed along the equi-sample-size contour. There are cases where we have unbalanced panels, which have differing numbers of time series observations across cross-sectional units. In most cases, however, all the cross-sectional units have the same time series dimension. To implement our tests based on the equi-squared-sum asymptotics to such panels, we may need to generate additional observations for some cross-sections (which have deficient sums of squared variations), or discard a part of the available observations for other cross-sections (which have overly sufficient sums of squared variations). The generation of the additional data may simply be done using the standard bootstrap procedure for the unit root models. The procedure creates the pseudo-data whose distributions are asymptotically identical to the original data, and have the required level of squared variations. Using the pseudo-data or discarding some portions of the real data may adversely affect the performances of the tests. They may distort, at least to some degrees, the finite sample sizes and lower the powers of the tests. Our extensive simulation studies, however, show that the prices we have to pay here to reach the new contour are relatively small compared to the efficiency gain from the normal asymptotics of the individual tests. The tests based on the equi-squared-sum indeed perform significantly better than other existing tests.

There is one special case where two contours become identical. This is when the usual sign function is used as the instrument generating function. The resulting IV estima- 
tor, which is often called the Cauchy estimator, has the fixed sum of squared IV's given by the sample size for its all possible realizations. The Cauchy $t$-ratio therefore has the standard normal $T$-asymptotics, along the contours of both the equi-squared-sum and the equi-sample-size. This is rather important. As we discussed above, to do inference on a fixed size data along the contour of the equi-squared-sum necessarily requires that we should either rely on the bootstrapped pseudo-data or do not fully utilize the observed data. The Cauchy estimator is an exception here. For the Cauchy estimator, neither bootstrapping nor discarding the data is necessary. As Phillips, Park and Chang (2004) point out earlier, the Cauchy estimator is sub-optimal and expected to have powers lower than the OLS estimator. However, our simulation study makes it clear that the sub-optimality of the Cauchy estimator quickly becomes unimportant as the number of the cross-sectional units increases in panels. In most cases, the Cauchy $t$-ratio performs at least as good as the OLS $t$-ratio using the generated or partially discarded samples with the same sum of squares.

The rest of the paper is organized as follows. In Section 2, we derive the main results of the paper for the nonlinear IV unit root test. There we introduce the unit root model, the instrument generating functions and the test statistic, and develop new asymptotics for the nonlinear IV $t$-ratios along the contour of the fixed sum of squared IV's. In particular, the asymptotics for the nonlinear IV $t$-ratios are shown to be normal along the new contour. Section 3 presents a method of reaching the equi-squared-sum contour by bootstrap for samples with a fixed size. The asymptotics for the IV $t$-ratios computed using the extended samples are also given. Section 4 extends our main results into more general models, which have intercepts and higher order dynamics. It is shown that our main results continue to hold for such general models. Section 5 demonstrates that our normal asymptotics along the new equi-squared-sum contour may be exploited to construct tools and methodologies for effective inferences in nonstationary panels. Finite sample performances of the newly proposed tests are evaluated via a set of simulations in Section 6. The concluding remarks are given in Section 7, and the mathematical proofs are collected in Appendix.

A word on notation. As usual, $\rightarrow_{d}$ and $\rightarrow_{a . s .}$ are used to signify respectively the convergence in distribution and the almost sure convergence, and $\triangle$ denotes the usual difference operator.

\section{New Contour Asymptotics for Nonlinear IV t-Ratios}

In this section, we develop the new asymptotics for the nonlinear IV $t$-ratios along the contour of the fixed sum of squared IV's. To effectively deliver the main idea, we consider the simple AR(1) model here. Our theories will be extended later to more general $\operatorname{AR}(p)$ models and the models with intercept. All the models considered in this section are univariate. The panel models will be considered later in subsequent sections. The model we consider here is given by

$$
\triangle y_{t}=\beta y_{t-1}+\varepsilon_{t},
$$

where

$$
\beta=0
$$


and $\left(\varepsilon_{t}\right)$ are martingale differences satisfying the usual conditions for the unit root model as in, e.g., Chang and Park (2002). In particular, we assume that an invariance principle holds for $\left(\varepsilon_{t}\right)$, i.e., if we let

$$
W_{T}(r)=\frac{1}{\sqrt{T}} \sum_{t=1}^{[T r]} \varepsilon_{t}
$$

for $r \in \mathbb{R}$, then

$$
W_{T} \rightarrow{ }_{d} W
$$

where $W$ is Brownian motion with variance $\sigma^{2}$. The assumption for $\left(\varepsilon_{t}\right)$ made here will be maintained throughout the paper.

The unit root hypothesis (2) may be tested using various methods. Here we consider the test based on the nonlinear IV methodology introduced recently by Chang (2002) and Phillips, Park and Chang (2004). The nonlinear IV estimator of $\beta$ is defined by

$$
\hat{\beta}_{T}=\left(\sum_{t=1}^{T} y_{t-1} F\left(y_{t-1}\right)\right)^{-1} \sum_{t=1}^{T} F\left(y_{t-1}\right) \triangle y_{t},
$$

which uses $F\left(y_{t-1}\right)$ as an instrument constructed from some instrument generating function (IGF) $F: \mathbb{R} \rightarrow \mathbb{R}$. Therefore, if we let

$$
s\left(\hat{\beta}_{T}\right)=\sigma\left(\sum_{t=1}^{T} y_{t-1} F\left(y_{t-1}\right)\right)^{-1}\left(\sum_{t=1}^{T} F\left(y_{t-1}\right)^{2}\right)^{1 / 2}
$$

be the standard error of $\hat{\beta}_{T}$, the IV $t$-ratio can be written as

$$
R(T)=\frac{\hat{\beta}_{T}}{s\left(\hat{\beta}_{T}\right)} .
$$

Obviously, $\sigma$ is unknown and we should use its consistent estimate to practically implement the IV $t$-ratio $R_{T} .^{2}$ The replacement of $\sigma$ by any of its consistent estimates, however, does not affect our subsequent asymptotic analysis of the IV $t$-ratio. Note that the IV $t$-ratio $R(T)$ reduces to

$$
(1 / \sigma)\left(\sum_{t=1}^{T} F\left(y_{t-1}\right)^{2}\right)^{-1 / 2} \sum_{t=1}^{T} F\left(y_{t-1}\right) \varepsilon_{t}
$$

under the null hypothesis of a unit root.

In addition to the unit root null hypothesis given by (2), we also consider in the paper the local-to-unity alternative

$$
\beta=-\frac{\delta}{T}
$$

\footnotetext{
${ }^{2}$ Consistent estimates of the error variance can be easily obtained either from the OLS residuals or more generally from the IV residuals satisfying the validity conditions in Phillips, Park and Chang (2004).
} 
to investigate the power of the unit root tests under the near unit root models. It is well known that the asymptotics for the near unit root models involve the Ornstein-Uhlenbeck process given by

$$
W_{\delta}(r)=\int_{0}^{r} e^{-\delta(r-s)} d W(s)
$$

where $\delta$ is the locality parameter in (6) and $W$ is the limit Brownian motion given in (3). For the asymptotics of the near unit root models in general, see, e.g., Stock (1994). The Ornstein-Uhlenbeck process $W_{\delta}$ in (7), as well as the limit Brownian motion $W$ in (3), will appear frequently in our subsequent asymptotic results.

As shown earlier by Phillips, Park and Chang (2004), the asymptotic behavior of the IV $t$-ratio depends crucially on the type of the instrument generating function $F$, in particular on whether it is integrable or asymptotically homogeneous. Here we mainly consider the IV $t$-ratio based on asymptotically homogeneous IGF. The IV $t$-ratio with integrable IGF is thoroughly explored in Chang (2002) for the test of unit roots in panels with cross-sectional dependency. An asymptotically homogeneous function $F$ may be written as

$$
F(\lambda x)=\kappa(\lambda) H(x)+o(\kappa(\lambda))
$$

for large $\lambda$ uniformly in $x \in \mathbb{R}$ over any compact interval, where we call $\kappa$ the asymptotic order and $H$ the limit homogeneous function of $F$. The reader is referred to Park (2004) for more precise definitions of asymptotically homogeneous functions.

The following lemma presents the asymptotic distributions of the IV $t$-ratio under the unit root null and the local-to-unity alternative along the conventional contour.

Lemma 2.1 Under the null hypothesis (2) of a unit root, we have

$$
R(T) \rightarrow d \frac{1}{\sigma}\left(\int_{0}^{1} H(W(r))^{2} d r\right)^{-1 / 2} \int_{0}^{1} H(W(r)) d W(r)
$$

as $T \rightarrow \infty$. Moreover, under the local-to-unity alternative (6), we have

$$
R(T) \rightarrow_{d}-\delta A+B
$$

where

$$
A=\frac{\int_{0}^{1} W_{\delta}(r) H\left(W_{\delta}(r)\right) d r}{\sigma\left(\int_{0}^{1} H\left(W_{\delta}(r)\right)^{2} d r\right)^{1 / 2}}, \quad B=\frac{\int_{0}^{1} H\left(W_{\delta}(r)\right) d W(r)}{\sigma\left(\int_{0}^{1} H\left(W_{\delta}(r)\right)^{2} d r\right)^{1 / 2}}
$$

as $T \rightarrow \infty$.

The limiting null distribution of the IV $t$-ratio in Lemma 2.1 has already been derived by Phillips, Park and Chang (2004). It is included here for completeness. The limit distribution of the IV $t$-ratio with an asymptotically homogeneous IGF is generally nonnormal. 
The asymptotics for the IV $t$-ratio under the local-to-unity alternatives in Lemma 2.1 are newly developed here. Interestingly, the IV $t$-ratios defined with asymptotically homogeneous IGF's generally have nontrivial discriminatory powers against the local-to-unity alternatives, which shrink towards the null at the rate $T^{-1}$. To see this, notice first that

$$
\int_{0}^{1} W_{\delta}(r) H\left(W_{\delta}(r)\right) d r=\int_{-\infty}^{\infty} x H(x) L_{\delta}(1, x) d x
$$

where $L_{\delta}(r, x)$ is the local time of $W_{\delta}$ at time $r$ and spatial point $x$. This follows directly from the application of the well known occupation times formula. See Revez and Yor (1994) for formal definitions of the local time and the occupation times formula. As a consequence, the IV $t$-ratio has nontrivial powers a.s. under the local alternatives as long as

(i) $x H(x) \geq 0$ for all $x \in \mathbb{R}$, and

(ii) $x H(x)>0$ for all $x$ in some neighborhood of the origin.

These will be referred to as the IGF validity conditions. Recall that $L_{\delta}(1, \cdot)$ is nonzero a.s. in some neighborhood of the origin, since $W_{\delta}$ starts at the origin.

It is rather remarkable that all the IV $t$-ratios with valid asymptotically homogeneous IGF's have nontrivial discriminatory powers in the $T^{-1}$-neighborhood of the null. For the IV estimator $\hat{\beta}_{T}$ defined in (4) with the IGF $F$ that has asymptotic order $\kappa$ and limit homogeneous function $H$, we have under the null $\beta=0$

$$
\sqrt{T} \kappa(\sqrt{T}) \hat{\beta}_{T} \rightarrow_{d}\left(\int_{0}^{1} W(r) H(W(r)) d r\right)^{-1} \int_{0}^{1} H(W(r)) d W(r)
$$

as shown in Phillips, Park and Chang (2004), and, in particular, it follows that

$$
\hat{\beta}_{T}=O_{p}\left(\frac{1}{\sqrt{T} \kappa(\sqrt{T})}\right) .
$$

The convergence rate for $\hat{\beta}_{T}$ is therefore dependent upon the asymptotic order $\kappa$ of the IGF $F$. Any IV $t$-ratio with a valid asymptotically homogenous IGF, however, has nontrivial discriminating powers against the $T^{-1}$-local alternatives, regardless of the asymptotic order of the IGF.

Of course, the OLS estimator belongs to the class of the IV estimators considered here. It is easy to see that the OLS estimator is an IV estimator with the instrument generating function $F(x)=x$, i.e., the identity function. Phillips, Park and Chang (2004) show that the OLS estimator is indeed the most efficient IV estimator, and therefore the usual $t$-ratio based on the OLS estimator is expected to be most powerful. Along the conventional equisample-size contour, the usual OLS based $t$-ratio has the limiting null distribution that is often referred to as the Dickey-Fuller distribution.

Now we derive the asymptotics along the new contour given by the equi-squared-sum of the IV's. For this, we define

$$
S_{T}=\inf _{K \geq 1}\left\{\frac{1}{T \kappa^{2}(\sqrt{T})} \sum_{t=1}^{K} F\left(y_{t-1}\right)^{2} \geq c\right\}
$$


for some fixed constant $c \in \mathbb{R}$. For each $T, S_{T}$ is the stopping time for which the sum of squares of IV's reaches the level $c T \kappa^{2}(\sqrt{T})$. As shown in Park and Phillips (1999), we have

$$
\frac{1}{T \kappa^{2}(\sqrt{T})} \sum_{t=1}^{[T \cdot]} F\left(y_{t-1}\right)^{2} \rightarrow_{d} \int_{0}^{\cdot} H(W(r))^{2} d r
$$

as $T \rightarrow \infty$. Therefore, if we let $\tau(c)$ be the stopping time defined by

$$
\int_{0}^{\tau(c)} H(W(r))^{2} d r=c,
$$

then we may expect $S_{T} / T \rightarrow{ }_{d} \tau(c)$ as $T \rightarrow \infty$, jointly with the invariance principle (3).

Theorem 2.2 Under the null hypothesis (2) of a unit root, we have

$$
R\left(S_{T}\right) \rightarrow_{d} \mathbb{N}(0,1)
$$

as $T \rightarrow \infty$. Moreover, under the local-to-unity alternative (6), we have

$$
R\left(S_{T}\right) \rightarrow_{d}-\delta A^{\circ}+B^{\circ}
$$

where

$$
\begin{aligned}
& A^{\circ}=\frac{1}{\sqrt{c} \sigma} \int_{0}^{\tau(c)} W_{\delta}(r) H\left(W_{\delta}(r)\right) d r \\
& B^{\circ}=\frac{1}{\sqrt{c} \sigma} \int_{0}^{\tau(c)} H\left(W_{\delta}(r)\right) d W(r) \sim \mathbb{N}(0,1)
\end{aligned}
$$

as $T \rightarrow \infty$.

The asymptotics for the IV $t$-ratio along the contour given by the same sum of squared IV's extend the results in Chang and Park (2004) developed for the usual $t$-ratio based on the OLS estimator. Of course, our results in Theorem 2.2 reduce to theirs if we set $F(x)=x$. Under the null hypothesis of the unit root, the IV $t$-ratio has the normal asymptotics of the nonlinear IV $t$-ratios along the new contour of equi-squared-sum of IV's. This is in sharp contrast with the nonstandard and nonnormal asymptotics along the conventional equisample-size contour given in Lemma 2.1. Under the local alternatives, both asymptotics provide the IV $t$-ratios some nontrivial powers as long as they use IGF's satisfying the validity conditions introduced above.

A special case of particular interest arises if we set

$$
F(x)=\operatorname{sgn}(x) .
$$

The resulting IV estimator, called the Cauchy estimator, was investigated earlier by So and Shin (1999b). This is an interesting example where the two contours coincide. Obviously, we have $F\left(y_{t-1}\right)^{2} \equiv 1$ for all $t$, and $\kappa(\lambda)=1$ for $F$ given in (9). With the only conformable 
value $c=1$, we thus have in this case that $S_{T}=T$. The contours of the equi-samplesize and the equi-squared-sum of the IV's therefore become identical for any realization of the samples. Consequently, both the conventional approach and our new approach here yield the same normal asymptotics for the $t$-ratio constructed from the Cauchy estimator. Needless to say, we would have the same results for any IV estimator with asymptotically homogeneous $F$ having the sign function as the limit homogeneous function. Note that for all in this class of IV estimators, we have $x \operatorname{sgn}(x)=|x|$, and therefore, the IGF validity conditions are satisfied. This in turn implies that the IV $t$-ratios constructed from the IV estimators in this class would all have nontrivial powers against the local alternatives shrinking towards unity at the rate $T^{-1}$.

\section{Reaching the Contour by Bootstrap}

Now we discuss the method of reaching the equi-squared-sum contour by bootstrap. This method will be crucial in the construction of the panel unit root tests to be introduced in Section 5. For a fixed sample size $T$, the contour given by the equi-squared-sum of IV's may not be attainable. That is, there may be cases with $S_{T}>T$, where $S_{T}$ is the stopping time introduced in (8). In fact, for all instrument generating functions other than the sign function given in (9), there is nonzero probability that such cases arise for any value of $c>0$. To utilize our new asymptotics within the fixed $T$ framework, we therefore need to extend our samples. Here we consider the bootstrap method to achieve this goal. Of course, one may use other resampling methods for this purpose.

The bootstrap method we propose here is simple and straightforward, and can be done following the steps we suggest below. We suppose that $T$ time series observations, $y_{1}, \ldots, y_{T}$, are available, and for a given $c>0$, we have $T<S_{T}$. The case would arise whenever $\left(T / \kappa^{2}(\sqrt{T})\right)^{-1} \sum_{t=1}^{T} F\left(y_{t-1}\right)^{2}<c$, i.e., the normalized sum of squares of the IV's does not reach the preset level $c>0$.

Step 1: Obtain the fitted values $\left(\hat{\varepsilon}_{t}\right)$ of the innovations $\left(\varepsilon_{t}\right)$ in $(1)$.

Step 2: Draw the bootstrap samples $\left(\varepsilon_{t}^{*}\right)$ from the fitted residuals $\left(\hat{\varepsilon}_{t}\right)$, after centering them by subtracting their sample mean, i.e., from $\left(\hat{\varepsilon}_{t}-T^{-1} \sum_{t=1}^{T} \hat{\varepsilon}_{t}\right)$.

Step 3: Set

$$
y_{t}^{*}=y_{T}+\sum_{k=T+1}^{t} \varepsilon_{t}^{*}
$$

for $t=T+1, \ldots$, and generate the extended sample $\left(y_{t}^{+}\right)$as

$$
y_{t}^{+}= \begin{cases}y_{t} & \text { if } t \leq T \\ y_{t}^{*} & \text { if } t \geq T+1\end{cases}
$$

Define the stopping time $S_{T}^{+}$similarly as in (8) using the extended sample $\left(y_{t}^{+}\right)$.

It is quite clear that the extended time series $\left(y_{t}^{+}\right), t=1, \ldots, S_{T}^{+}$, behave similarly as the original full sample $\left(y_{t}\right), t=1, \ldots, S_{T}$, especially when $T$ is large. Note that the bootstrap 
samples used to obtain $\left(y_{t}^{*}\right), t=T+1, \ldots, S_{T}^{+}$, for extending the given sample are drawn from the empirical distribution of the innovations, which converges in distribution to their true underlying distribution as $T$ increases.

On the proposed bootstrap procedure, there are several issues that should be addressed here. First, we may use any IV estimator (including of course the OLS estimator) to estimate the parameter $\beta$ in Step 1. It is also allowed to set $\beta=0$ and use the first differences of $\left(y_{t}\right)$ as the fitted residulas $\left(\hat{\varepsilon}_{t}\right)$ in this step. In fact, neither the choice of the IV estimator nor the imposition (or non-imposition) of the unit root restriction affects any of our asymptotic results established in the paper. Second, note that we deliverately impose the unit root in our generation of the extended data $\left(y_{t}^{*}\right)$ from the bootstrap samples $\left(\varepsilon_{t}^{*}\right)$ in Step 3. This is important and crucial for the validity of the unit root testing considered in the paper. Indeed, it is well known that the bootstrap for the unit root models is generally inconsistent if the presence of unit root is not imposed in generating bootstrap samples. See Basawa et al. (1991).

Throughout the paper, we denote by $\left(y_{t}^{+}\right)$the sample of size $S_{T}^{+}$extended by the bootstrap procedure introduced above. By convention, we continue to use the same notation when $S_{T} \leq T$, in which case we have $S_{T}^{+} \equiv S_{T}$ and $y_{t}^{+} \equiv y_{t}$ for all $t=1, \ldots S_{T}$. Moreover, we signify by $R\left(S_{T}^{+}\right)$the IV $t$-ratio computed from $\left(y_{t}^{+}\right)$for the sample of size $S_{T}^{+}$. Once again, we have $R\left(S_{T}\right) \equiv R\left(S_{T}^{+}\right)$if $S_{T} \leq T$. Now we present the asymptotics for the extended IV $t$-ratio $R\left(S_{T}^{+}\right)$constructed from the extended sample $\left(y_{t}^{+}\right)$. We first define the limit processes apprearing in our new limit theory. Corresponding to the BM $W$ defined in (3), we define $W^{+}$for the extended sample by

$$
W^{+}(r)=\left\{\begin{array}{ll}
W(r) & \text { if } r \leq 1 \\
W(1)+W^{*}(r-1) & \text { if } r>1
\end{array},\right.
$$

where $W^{*}$ is a $\mathrm{BM}$ independent of $W(r)$ for $r \in[0,1]$. It is easy to see that $W^{+}$is a $\mathrm{BM}$ itself. Moreover, in place of the Ornstein-Uhlenbeck process $W_{\delta}$ introduced in (7), we let $W_{\delta}^{+}$be the process given by

$$
W_{\delta}^{+}(r)=\left\{\begin{array}{ll}
W_{\delta}(r) & \text { if } r \leq 1 \\
W_{\delta}(1)+W^{*}(r-1) & \text { if } r>1
\end{array} .\right.
$$

Again, the BM $W^{*}$ is independent of $W_{\delta}(r)$ for $r \in[0,1]$, since it is independent of $W(r)$ which generates $W_{\delta}(r)$ for $r \in[0,1]$. One may easily note that $W_{\delta}^{+}$is an Ornstein-Uhlenbeck process driven by the Brownian motion $W$ up to the time unity, and then becomes the process having Brownian increments independent of the driving Brownian motion $W$ afterwards.

We may now deduce that

Corollary 3.1 Under the null hypothesis (2) of a unit root, we have

$$
R\left(S_{T}^{+}\right) \rightarrow_{d} \mathbb{N}(0,1)
$$

as $T \rightarrow \infty$. Moreover, under the local-to-unity alternative (6), we have

$$
R\left(S_{T}^{+}\right) \rightarrow_{d}-\delta A^{+}+B^{+},
$$


where

$$
\begin{aligned}
& A^{+}=\frac{1}{\sqrt{c} \sigma} \int_{0}^{1 \wedge \tau(c)} W_{\delta}(r) H\left(W_{\delta}(r)\right) d r \\
& B^{+}=\frac{1}{\sqrt{c} \sigma} \int_{0}^{\tau(c)} H\left(W_{\delta}^{+}(r)\right) d W^{+}(r) \sim \mathbb{N}(0,1)
\end{aligned}
$$

as $T \rightarrow \infty$.

It is now clearly seen from Corollary 3.1 that the IV $t$-ratio $R\left(S_{T}^{+}\right)$obtained using the extended sample has the standard normal limiting distribution, exactly as for the original IV $t$-ratio $R\left(S_{T}\right)$ defined on the new contour. Moreover, it can be readily deduced from Corollary 3.1 that $R\left(S_{T}^{+}\right)$has nontrivial powers against the local-to-unity alternatives.

The powers of the extended IV $t$-ratio $R\left(S_{T}^{+}\right)$, however, will be smaller than the original IV $t$-ratio $R\left(S_{T}\right)$ defined on the new contour, if $T<S_{T}$ and the contour is indeed attained via the extension of the sample by the bootstrap. This can be well expected, since the extended bootstrap samples are obtained under the null hypothesis of the unit root. This reduction in the power of $R\left(S_{T}^{+}\right)$relative to $R\left(S_{T}\right)$ can be easily seen from our results in Theorem 2.2 and Corollary 3.1. Note that $1<\tau(c)$ in this case, and we have $x H(x) \geq 0$. Consequently, it follows that $A^{\circ} \geq A^{+}$, which implies that $R\left(S_{T}\right)$ has a greater mean shift effect than $R\left(S_{T}^{+}\right)$under the local-to-unity alternatives. It is therefore unambiguous that the former is more powerful than the latter under the local-to-unity alternatives. Note however that the test $R\left(S_{T}\right)$ is infeasible as it requires more data than are available.

On the other hand, it is not possible to unambiguously compare the extended IV $t$-ratio $R\left(S_{T}^{+}\right)$on the new contour with the fixed sample IV $t$-ratio $R(T)$ defined on the conventional contour in terms of the powers against the local-to-unity alternatives. Recall that both tests utilize the given samples of size $T$, so they are both feasible in the usual cases with fixed $T$. Thus comparing the two may shed light on the effect of attaining the new contour by bootstrap. Their asymptotics under the local-to-unity alternatives, which are respectively given in Lemma 2.1 and Corollary 3.1, show that none of them indeed dominates the other. To see this, let $T<S_{T}$ and $1<\tau(c)$. Then it necessarily follows that $A \geq A^{+}$, and this implies that $R(T)$ has a greater mean shift effect than $R\left(S_{T}^{+}\right)$incurred by the presence of the locality parameter $\delta$. However, the additional terms $B$ and $B^{+}$independent of $\delta$ render the comparison in the opposite direction. While $B^{+}$is the standard normal and in particular distributed symmetrically, $B$ has a nonstandard distribution that is skewed to the left towards the alternative distributions. This would obviously affect the powers of $R(T)$ adversely, compared to $R\left(S_{T}^{+}\right)$. The local power comparison between $R\left(S_{T}^{+}\right)$and $R(T)$ would therefore be ultimately dependent upon the magnitude of the locality parameter $\delta$. It is likely that the former is more powerful if $\delta$ is small, while the comparison may be reversed as $\delta$ takes a larger value. 


\section{Extensions to General Models}

All our previous results may be easily and naturally extended to more general unit root models and the models with intercept under some simple and obvious modifications. ${ }^{3} \mathrm{We}$ now consider the model

$$
y_{t}=\mu+y_{t}^{\circ},
$$

where $\left(y_{t}^{\circ}\right)$ follows an $\operatorname{AR}(p)$ process specified as

$$
\triangle y_{t}^{\circ}=\beta y_{t-1}^{\circ}+\sum_{k=1}^{p-1} \alpha_{k} \triangle y_{t-k}^{\circ}+\varepsilon_{t}
$$

We let $\beta=0$ and $\left(\varepsilon_{t}\right)$ be martingale differences as in (1). The model given in (12) will subsequently be referred to simply as the model with intercept. Throughout the paper, we assume that the standard assumptions imposed on the unit root models hold for the AR process in (13). The reader is referred to, for instance, Chang and Park (2002), for the detailed conditions that we require here.

The unit root hypothesis $\beta=0$ for the general models introduced in (12) and (13) can be tested in the regression

$$
\triangle y_{t}=\beta \tilde{y}_{t-1}+\sum_{k=1}^{p-1} \alpha_{k} \triangle y_{t-k}+\varepsilon_{t},
$$

where $\left(\tilde{y}_{t}\right)$ is given by

$$
\tilde{y}_{t}=y_{t}-y_{1}
$$

or

$$
\tilde{y}_{t}=y_{t}-\frac{1}{t} \sum_{k=1}^{t} y_{k} .
$$

Note that $\left(\tilde{y}_{t}\right)$ 's in (15) and (16) both are invariant with respect to the intercept term $\mu$ introduced in (12). The demeaning procedures in (15) and (16) rely only on the past observations, and this is crucial for our testing procedures developed in the paper. Roughly, they allow the demeaned series to weakly converge to martingales, which is necessary for our normal asymptotic theories to hold.

The nonlinear IV $t$-ratio can be easily defined for regression (14), where we use

$$
\left(F\left(\tilde{y}_{t-1}\right), \triangle y_{t-1}, \ldots, \triangle y_{t-p+1}\right)^{\prime}
$$

as the instruments. Here either $\left(\tilde{y}_{t}\right)$ defined in (15) or the one given in (16) may be used. Of course, the IV $t$-ratio for $\beta$ in regression (14) can be defined similarly as in (5). The

\footnotetext{
${ }^{3}$ The models with linear time trend can also be dealt with, once we come up with a recursive detrending scheme which ensures the martingale property of the detrended data. The implementation of our methodology is then rather straightforward, given our results for the models with intercept obtained in this section.
} 
reader is referred to Chang (2002), for the explicit formula for the IV $t$-ratio in this general case. Naturally, the new contour is given by

$$
\tilde{S}_{T}=\inf _{K \geq 1}\left\{\frac{1}{T \kappa^{2}(\sqrt{T})} \sum_{t=1}^{K} F\left(\tilde{y}_{t-1}\right)^{2} \geq c\right\}
$$

for some fixed constant $c \in \mathbb{R}$, similarly as in (8).

In what follows, we denote by $\tilde{R}(T)$ and $R\left(\tilde{S}_{T}\right)$ the IV $t$-ratios for $\beta$ in regression (14) respectively on the conventional contour with fixed sample size and along the new contour given by (17). Also, we let

$$
\alpha(1)=1-\sum_{k=1}^{p-1} \alpha_{k},
$$

where $\left(\alpha_{k}\right)$ are the AR coefficients in our model (13), and define Brownian motion $V$ by

$$
V(r)=\alpha(1)^{-1} W(r)
$$

and the corresponding Ornstein-Uhlenbeck process $V_{\delta}$ by

$$
V_{\delta}(r)=\int_{0}^{r} e^{-\delta(r-s)} d V(s)
$$

similarly as in (7).

Corollary 4.1 Suppose that the demeaning procedure in (15) is used. We have under the null hypothesis (2) of a unit root,

$$
\begin{aligned}
\tilde{R}(T) & \rightarrow{ }_{d} \frac{1}{\sigma}\left(\int_{0}^{1} H(V(r))^{2} d r\right)^{-1 / 2} \int_{0}^{1} H(V(r)) d W(r) \\
R\left(\tilde{S}_{T}\right) & \rightarrow{ }_{d} \mathbb{N}(0,1)
\end{aligned}
$$

as $T \rightarrow \infty$, and under the local-to-unity alternative (6)

$$
\begin{gathered}
\tilde{R}(T) \rightarrow_{d}-\delta \tilde{A}+\tilde{B} \\
R\left(\tilde{S}_{T}\right) \rightarrow_{d}-\delta \tilde{A}^{\circ}+\tilde{B}^{\circ}
\end{gathered}
$$

as $T \rightarrow \infty$, where $(\tilde{A}, \tilde{B})$ and $\left(\tilde{A}^{\circ}, \tilde{B}^{\circ}\right)$ are defined similarly as $(A, B)$ and $\left(A^{\circ}, B^{\circ}\right)$, given respectively in Lemma 2.1 and Theorem 2.2 , only with $W_{\delta}$ replaced by $V_{\delta}$. As earlier, $\tilde{B}^{\circ}$ is distributed as standard normal.

We now define the stochastic processes $\tilde{V}$ and $\tilde{V}_{\delta}$ by

$$
\begin{aligned}
& \tilde{V}(r)=V(r)-\frac{1}{r} \int_{0}^{s} V(s) d s \\
& \tilde{V}_{\delta}(r)=V_{\delta}(r)-\frac{1}{r} \int_{0}^{s} V_{\delta}(s) d s
\end{aligned}
$$


for $r \in(0, \infty)$ and let $\tilde{V}(0)=\tilde{V}_{\delta}(0)=0$. Then $\tilde{V}$ and $\tilde{V}_{\delta}$ become well defined continuous stochastic processes on $[0, \infty) .{ }^{4}$ The limit theories for the IV $t$-ratios under the recursive demeaning scheme (16) are represented by the processes $\tilde{V}$ and $\tilde{V}_{\delta}$, respectively for the null and the alternative limit distributions. We have

Corollary 4.2 Suppose that the demeaning procedure in (16) is used. We have under the null hypothesis (2) of a unit root,

$$
\begin{aligned}
\tilde{R}(T) & \rightarrow{ }_{d} \frac{1}{\sigma}\left(\int_{0}^{1} H(\tilde{V}(r))^{2} d r\right)^{-1 / 2} \int_{0}^{1} H(\tilde{V}(r)) d W(r) \\
R\left(\tilde{S}_{T}\right) & \rightarrow{ }_{d} \mathbb{N}(0,1)
\end{aligned}
$$

as $T \rightarrow \infty$, and under the local-to-unity alternative (6),

$$
\begin{aligned}
\tilde{R}(T) & \rightarrow_{d}-\delta \tilde{A}+\tilde{B} \\
R\left(\tilde{S}_{T}\right) & \rightarrow_{d}-\delta \tilde{A}^{\circ}+\tilde{B}^{\circ}
\end{aligned}
$$

as $T \rightarrow \infty$, where $(\tilde{A}, \tilde{B})$ and $\left(\tilde{A}^{\circ}, \tilde{B}^{\circ}\right)$ are defined similarly as $(A, B)$ and $\left(A^{\circ}, B^{\circ}\right)$, given respectively in Lemma 2.1 and Theorem 2.2 , but now with $W_{\delta}$ replaced by $\tilde{V}_{\delta}$. As earlier, $\tilde{B}^{\circ}$ is distributed as standard normal.

Our results in Corollaries 4.1 and 4.2 imply that all our previous results essentially carry through more general models we introduce in (12) and (13). In particular, the distribution theories along the new contour are normal, if the demeaning procedure given in (15) or (16) is used.

The bootstrap procedure to attain the contour for the fixed sum of squared IV's can also be easily developed for the general models we consider here.

Step 1: Fit regression (14) and obtain the parameter estimates $\hat{\beta}$ and $\left(\hat{\alpha}_{k}\right)$ and the fitted values $\left(\hat{\varepsilon}_{t}\right)$ of the innovations $\left(\varepsilon_{t}\right)$ in $(13)$.

Step 2: Draw the bootstrap samples $\left(\varepsilon_{t}^{*}\right)$ from the centered fitted residuals $\left(\hat{\varepsilon}_{t}-T^{-1} \sum_{t=1}^{T} \hat{\varepsilon}_{t}\right)$.

Step 3: Construct the bootstrap sample $\left(\triangle y_{t}^{*}\right)$ for $t=T+1, \ldots$ using the regression model (14) with the estimated parameters $\hat{\beta}$ and $\left(\hat{\alpha}_{k}\right)$, and the bootstrap samples $\left(\varepsilon_{t}^{*}\right)$ for the innovations.

Step 4: Set

$$
\tilde{y}_{t}^{*}=\tilde{y}_{T}+\sum_{k=T+1}^{t} \triangle y_{t}^{*}
$$

for $t=T+1, \ldots$, and generate the extended sample $\left(\tilde{y}_{t}^{+}\right)$as

$$
\tilde{y}_{t}^{+}= \begin{cases}\tilde{y}_{t} & \text { if } t \leq T \\ \tilde{y}_{t}^{*} & \text { if } t \geq T+1\end{cases}
$$

Define the stopping time $\tilde{S}_{T}^{+}$similarly as in (8) using the extended sample $\left(\tilde{y}_{t}^{+}\right)$.

\footnotetext{
${ }^{4}$ See Chang and Park (2004).
} 
As we mentioned earlier in Section 3, we may use OLS or any other IV estimator to estimate the model parameters and the fitted residuals in Step 1. Moreover, we may or may not impose the unit root restriction $\beta=0$ in this step. Similarly, it does not affect our subsequent results whether or not we impose the restriction $\beta=0$ in Step 3 to obtain the bootstrap samples $\left(\triangle y_{t}^{*}\right)$. In our simulations reported in Section 6 below, we use the simple OLS method to obtain the fitted residuals. For the parameter estimates, we rely on the restricted OLS, i.e., we let $\beta=0$ and estimate the other parameters $\left(\alpha_{k}\right)$ by the OLS method. The unit root is thus imposed for the parameter estimation in Steps 1 and 3 in the simulations conducted in the paper.

We now denote by $R\left(\tilde{S}_{T}^{+}\right)$the IV $t$-ratio computed from the extended sample $\left(y_{t}^{+}\right)$. Also, we let $V^{+}$and $V_{\delta}^{+}$be defined similarly as in (10) and (11) from $V, V_{\delta}$ and $V^{*}$, which is the Brownian motion independent of $V$ and has the same variance as $V$. Moreover, we signify by $\tilde{V}_{\delta}^{+}$the process that is given by $\tilde{V}_{\delta}$, defined in (21), up to time unity and becomes $V^{*}$ thereafter. Then we have

Corollary 4.3 Under the hypothesis (2) of a unit root, we have

$$
R\left(\tilde{S}_{T}^{+}\right) \rightarrow{ }_{d} \mathbb{N}(0,1)
$$

as $T \rightarrow \infty$. Moreover, we have under the local-to-unity alternative (6) that

$$
R\left(\tilde{S}_{T}^{+}\right) \rightarrow_{d}-\delta \tilde{A}^{+}+\tilde{B}^{+}
$$

as $T \rightarrow \infty$. If the demeaning procedure in (15) is used, then $\left(\tilde{A}^{+}, \tilde{B}^{+}\right)$are defined as $\left(A^{+}, B^{+}\right)$given in Corollary 3.1, with $W_{\delta}$ and $W_{\delta}^{+}$replaced by $V_{\delta}$ and $V_{\delta}^{+}$, respectively. On the other hand, if the demeaning procedure in (16) is used, then $\left(\tilde{A}^{+}, \tilde{B}^{+}\right)$are defined as $\left(A^{+}, B^{+}\right)$with $W_{\delta}$ and $W_{\delta}^{+}$replaced by $\tilde{V}_{\delta}$ and $\tilde{V}_{\delta}^{+}$, respectively.

Once again, the limit theories for the IV $t$-ratio for the extended samples in more general models given by (12) and (13) are virtually identical to the results for the simple model (1) considered in Section 2.

If we let the AR order $p$ in (14) increase as the sample size gets large, the unit root test that we consider here is valid for more general processes than the $\operatorname{AR}(p)$ model in (13). This is now well known. It was first noted by Said and Dickey (1984), who show that the test based on the standard $t$-ratio is valid for general invertible ARMA models of unknown orders if we increase the order $p$ of the fitted autoregression by $p=c T^{k}$ with some constant $c>0$ and the rate of expansion $k \in(0,1 / 3]$. More recently, Chang and Park (2002) show that the procedure is indeed valid for more general linear processes with minimal summability conditions on their coefficients and under much weaker condition $p=o\left(T^{1 / 2}\right)$ on the rate of increase for the fitted AR orders. It can be shown that the result by Chang and Park (2002) continue to hold for the tests based on the nonlinear IV's along the new contour. Therefore, our result here is applicable for a broad range of time series models. 


\section{Panel Unit Root Tests}

We now consider a panel unit root model given by

$$
y_{i t}=\mu_{i}+y_{i t}^{\circ}
$$

and

$$
\triangle y_{i t}^{\circ}=\beta y_{i, t-1}^{\circ}+\sum_{k=1}^{p_{i}-1} \alpha_{i k} \triangle y_{i, t-k}^{\circ}+\varepsilon_{i t}
$$

with

$$
\beta_{i}=0
$$

for $i=1, \ldots, N$ and $t=1, \ldots, T$. As usual, the index $i$ denotes individual cross-sectional units, such as individuals, households, industries or countries, and the index $t$ denotes time periods. Note that we allow for heterogeneous individual effects and shortrun dynamics here. For each cross-section $i=1, \ldots, N$, the model given by (22) and (23) is precisely what we introduced in (12) and (13). As before, we assume that the error terms $\left(\varepsilon_{i t}\right)$ are martingale differences. Moreover, for the moment, we let $\left(\varepsilon_{i t}\right)$ be uncorrelated across crosssectional units. We make this simplifying assumption to concentrate better on important aspects of our new methodology. The cross-sectional dependency can be allowed and easily dealt with in our framework. This will be discussed later in more detail along with other issues in panel unit root tests.

Given all our results established in earlier sections, it is rather straightforward to develop the unit root test for testing the hypothesis (24) in our panel model given by (22) and (23). We consider the regression

$$
\triangle y_{i t}=\beta_{i} \tilde{y}_{i, t-1}+\sum_{k=1}^{p_{i}-1} \alpha_{i k} \triangle y_{i, t-k}+\varepsilon_{i t},
$$

which corresponds to the regression given in (14) for each cross-section $i=1, \ldots, N$. To introduce the approach relying on the new contour, we then let for each $i=1, \ldots, N$, as in (17), $S_{i} \geq 1$ be such that

$$
S_{i}=\inf _{K \geq 1}\left\{\frac{1}{T \kappa^{2}(\sqrt{T})} \sum_{t=1}^{K} F\left(\tilde{y}_{i, t-1}\right)^{2} \geq c\right\}
$$

for some fixed constant $c>0$, consider the nonlinear IV $t$-ratio $R\left(S_{i}\right)$ for the sample of size $S_{i}$. Here the sample size $S_{i}$ is determined by the squared sum of the lagged instrument $F\left(\tilde{y}_{i, t-1}\right)$ achieving a certain level. Note that $S_{i}$ is also given as a function of $T$, but its dependency on $T$ is suppressed for expositional brevity. Of course, we may have to resort to the bootstrap method to achieve the given contour, in which case we consider the individual IV $t$-ratios computed using the extended sample $\left(y_{i t}^{+}\right)$that can be constructed following the steps outlined in Section 4, for each cross-section $i=1, \ldots, N$. We will denote the test computed from the extended sample by $R\left(S_{i}^{+}\right)$analogously as in Section 4 . 
Under the null hypothesis (24), it follows immediately from Corollaries 4.2 and 4.3 that

$$
R\left(S_{i}\right), R\left(S_{i}^{+}\right) \rightarrow_{d} \mathbb{N}(0,1)
$$

as $T \rightarrow \infty$, for all $i=1, \ldots, N$. Here we have standard normal limit theory for the individual IV $t$-ratios from all cross-sections. Furthermore, it is quite obvious that they become independent across $i=1, \ldots, N$, since we assume that the innovations are crosssectionaly uncorrelated. The normal asymptotics here rely on $T \rightarrow \infty$, and therefore require the time series dimension $T$ to be large. The asymptotic normality and independence of the individual IV $t$-ratios are indeed what make them an efficient basis for constructing our panel unit root tests, as we shall see below.

Now suppose we are interested in testing whether the series $\left(y_{i t}\right)$ generated as in (22) and (23) has a unit root in all cross-sections $i=1, \ldots, N$, against the alternative that $\left(y_{i t}\right)$ are stationary in all cross-section $i$. The null hypothesis is therefore formulated as $\mathrm{H}_{0}: \beta_{i}=0$ for all $i$, and tested against the stationarity alternative $\mathrm{H}_{1}: \beta_{i}<0$ for all $i$. The test statistic we first consider for testing the panel unit root hypothesis is a simple average of the individual IV $t$-ratio statistics, $R\left(S_{i}\right)$ or $R\left(S_{i}^{+}\right)$, for testing $\beta_{i}=0$ computed from the regression (25) for each cross-sectional unit $i=1, \ldots, N$. The tests are defined as

$$
\bar{R}=\frac{1}{\sqrt{N}} \sum_{i=1}^{N} R\left(S_{i}\right), \quad \bar{R}^{+}=\frac{1}{\sqrt{N}} \sum_{i=1}^{N} R\left(S_{i}^{+}\right) .
$$

We note that no additional standardizations are applied to the individual tests $R\left(S_{i}\right)$ and $R\left(S_{i}^{+}\right)$in forming the average panel tests $\bar{R}$ and $\bar{R}^{+}$. This is because the individual IV $t$-tests, $R\left(S_{i}\right)$ and $R\left(S_{i}^{+}\right)$, themselves are already standard normal asymptotically, not requiring any further normalization. This is in sharp contrast to all the existing panel unit root tests, which require some sort of additional standardizations, such as mean and variance adjustments or $p$-value transformations, to derive standard limit theories. Our panel unit root tests, $\bar{R}$ and $\bar{R}^{+}$, have standard normal limit theories, which follow immediately from (27).

Theorem 5.1 Under the hypothesis (24) of unit roots, we have

$$
\bar{R}, \bar{R}^{+} \rightarrow_{d} \mathbb{N}(0,1)
$$

as $T \rightarrow \infty$ for all $N \geq 1$.

Our normal limit theory here is derived using $T$-asymptotics only, and the factor $N^{-1 / 2}$ in the definition of the test statistic $\bar{R}$ or $\bar{R}^{+}$in (28) is used just as a normalization factor, since the test, $\bar{R}$ or $\bar{R}^{+}$, is based on the sum of $N$ asymptotically independent random variables. This implies that the dimension of the cross-sectional units $N$ may take any value, small as well as large.

Our methodology has some important advantages over the existing tests. In our approach, the individual IV $t$-ratios are normal and independent across individual units as long as $T$ tends to infinity. This gives us the flexibility to fully investigate the presence 
of the unit roots in panels using the order statistics. For instance, we may use the minimum of $R\left(S_{i}\right)$ or $R\left(S_{i}^{+}\right)$across $i$ to test the null hypothesis $\mathrm{H}_{0}: \beta_{i}=0$ for all $i$ against the alternative $\mathrm{H}_{1}: \beta_{i}<0$ for some $i$. More interestingly, we may test the composite null hypothesis $\mathrm{H}_{0}: \beta_{i}=0$ for $1 \leq i \leq M$ with $0<M \leq N$, against the alternative $\mathrm{H}_{1}: \beta_{i}<0$ for all $i$, by using the maximum of $R\left(S_{i}\right)$ or $R\left(S_{i}^{+}\right)$across $i$. Let $M \bar{R}=\max _{1 \leq i \leq N} R\left(S_{i}\right), M \bar{R}^{+}=\max _{1 \leq i \leq N} R\left(S_{i}^{+}\right)$, and also define $m \bar{R}=\min _{1 \leq i \leq N} R\left(S_{i}\right)$ and $m \bar{R}^{+}=\min _{1 \leq i \leq N} R\left(S_{i}^{+}\right)$. Since $R\left(S_{i}\right)$ and $R\left(S_{i}^{+}\right)$are in the limit nothing but independent standard normal random variates, the limit theories for these order statistics can be easily obtained as in Chang and Song (2002). For a $\lambda$-level test, the critical values for the maximum statistics, $M \bar{R}$ and $M \bar{R}^{+}$, are given by $\Phi^{-1}\left(\lambda^{1 / M}\right)$ and those for the minimum statistics, $m \bar{R}$ and $m \bar{R}^{+}$, by $\Phi^{-1}\left(1-(1-\lambda)^{1 / N}\right)$, where $\Phi^{-1}$ is the inverse cummulative distribution function of a standard normal random variable. See Chang and Song (2002) for more details. Moreover, our normal asymptotics for the individual tests make it much easier to deal with the cross-sectional dependencies, which are known to be extremely difficult to control using the conventional approaches relying on nonnormal $T$-asymptotics.

The normal limit theory is also obtained for the existing panel unit root tests, such as the pooled OLS test by Levin, Lin and Chu (2002) and the group mean $t$-bar statistic by Im, Pesaran and Shin (2003); however, their tests involve the mean and variance adjustments for the individual tests. Moreover, their theories are applicable only for large $N$, and also require cross-sectional independence. More recently, several authors have made serious attempt to allow for cross-sectional dependencies. Chang (2004) allows for dependencies of unrestricted form, but her bootstrap procedure requires the dimension of time series $T$ to be substantially larger than that of the cross-section $N$, which is restrictive for many practical applications. On the other hand, the procedures by Choi (2001b), Phillips and Sul (2001), Moon and Perron (2001) and Bai and Ng (2004) allow for cross-sectional dependencies, but for those in some specific forms driven by common factors. Finally, Chang (2002) and Chang and Song (2002) uses the nonlinear IV approach to invent the tests for panel unit roots that are valid in the presence of arbitrary cross-sectional correlations among the innovations. Their limit theories are also normal.

Our framework is flexible enough to accommodate virtually all ingredients of previous researches. The nonlinear IV methods with integrable IGF's explored in Chang (2002) can be implemented here to deal with cross-sectional correlations among innovations in arbitrary forms. Moreover, the factor models for the cross-sectional dependencies employed in Choi (2001b), Phillips and Sul (2001), Moon and Perron (2001), and Bai and Ng (2002) can also be used together with our novel approach here. Once the cross-section dependency is dealt with by using one of the methods listed above, our panel tests continue to yield normal asymptotics without having to require the mean and variance adjustments or any other transformations for the individual tests. We actually investigated through simulations the performances of our tests for the panel models with cross-sectional dependency generated by common factors. It appears that the tests perform quite well in finite samples even for the panels with strong cross-section dependencies generated by common factors, once they are removed using a defactoring method such as the orthogonalization procedure suggested by Phillips and Sul (2001). This is true for the panels with various combinations of time and cross-sectional dimensions. 


\section{Simulations and Guidelines for Practical Implementations}

We evaluate the finite sample performance of the panel unit root test $\bar{R}^{+}$defined in (28) for testing the joint unit root hypotheses, which is the average of individual IV $t$-ratios computed along the new contour. We also examine the performance of the maximum statistics $M \bar{R}^{+}$for testing the composite null hypothesis that only some cross-sections have unit roots. For the simulations we consider two instrument generating functions, $F(x)=x$ and $\operatorname{sgn}(x)$, and use the resulting IV estimators, namely the OLS and Cauchy estimators, to construct our panel unit root tests. As discussed earlier, the individual Cauchy $t$-ratios are always on the equi-squared-sum contour, which is also the equi-sample-size contour, without any sample adjustment. However, we need to adjust the given sample size for each individual cross-section to ensure that the individual OLS $t$-ratios are on the equi-squared sum contour. We will discuss this issue of reaching the contour for the OLS $t$-ratios in more detail later, along with some other issues that may arise in the practical implementations of our new procedure.

We specify the simulation model as

$$
y_{i t}=\mu_{i}+y_{i t}^{\circ}
$$

and let the stochastic component $y_{i t}^{\circ}$ be generated as

$$
\triangle y_{i t}^{\circ}=\beta_{i} y_{i, t-1}^{\circ}+u_{i t},
$$

where

$$
\begin{aligned}
& u_{i t}=\rho_{i} u_{i, t-1}+v_{i t} \\
& v_{i t}=\pi_{i} w_{t}+\varepsilon_{i t}
\end{aligned}
$$

and $\left|\rho_{i}\right|<1,\left(w_{t}\right)$ and $\left(\varepsilon_{i t}\right)$ are independent and identically distributed random sequences. Under our specification, the stochastic component $\left(y_{i t}^{\circ}\right)$ of $\left(y_{i t}\right)$ has a unit root when $\beta_{i}=0$.

Our simulation model is simple, yet it is general enough to consider various important aspects of panel models that would affect the finite sample performance of the panel unit root tests. Our model allows for the individual fixed effects parametrized as $\left(\mu_{i}\right)$, the heterogeneous serial correlation structures given by the autoregressive coefficients $\left(\rho_{i}\right)$, and the presence of a common factor $\left(w_{t}\right)$ generating cross-sectional dependency with the heterogeneous factor loading coefficients $\left(\pi_{i}\right)$. The factor structure has been routinely used to generate cross-sectional dependency, see Bai and Ng (2004), Moon and Perron (2001) and Phillips and Sul (2001). We consider the following two cases:

\begin{tabular}{llc}
\hline & \multicolumn{1}{c}{ Cases } & Specifications \\
\hline (a) & Independent Case & $\pi_{i}=0$ \\
(b) & Dependent Case & \\
\hline
\end{tabular}


The above cases are considered to demonstrate that our methodology really works as the theory predicts, i.e., that along the new contour the individual tests can be regarded as independent standard normals and our average panel unit root tests $\bar{R}^{+}$and the maximum tests $M \bar{R}^{+}$are distributed approximately as standard normal and functional of cummulative standard normal distribution function, respectively. The independent case looks at the independent panels with no common factor, and the dependent case explores the general panels with cross-sectional dependency generated by the common factor.

The simulation results are reported in Tables 1-4. All the reported simulation results are based on 10,000 iterations. Panels with dimensions $N=10,50,100,200$ and $T=$ 25, 50, 100 are considered for the average statistics in Tables 1 and 2. A larger sample size $T=200$ is also considered for the maximum statistics in Tables 3 and 4 . For both the independent and dependent cases, $\left(\varepsilon_{i t}\right)$ are generated as independent standard normals, and the autoregressive coefficients $\left(\rho_{i}\right)$ are randomly drawn from the uniform distribution with support $[0.2,0.4]$. For the dependent case, the common factor $\left(w_{t}\right)$ is generated from the standard normal distribution and the factor loading coefficients $\left(\pi_{i}\right)$ on the common factor are also randomly drawn from the uniform distribution with support $[1,4]$, providing ample heterogeneity among the individual responsiveness to the common factor.

For the unit root null hypothesis, we set the coefficients $\beta_{i}=0$ for all $i=1, \ldots, N$, and investigate the finite sample sizes relative to the nominal 1\%, 5\% and $10 \%$ test sizes. To examine the rejection probabilities and the size-adjusted powers, we consider the stationary alternatives with $\left(\beta_{i}\right)$ generated randomly from the uniform distribution with support $[-0.05,0]$. We consider the $t$-ratios based on the OLS and the Cauchy estimators along the new contour, and call the panel unit root tests based on the OLS $t$-ratios the CP tests and those based on the Cauchy $t$-ratios the Cauchy tests. For comparison purpose in the independent models, we also consider the usual average $t$-ratio test along the conventional contour with the mean and variance corrections as done by Im, Pesaran and Shin (2003), which we denote by IPS. For the dependent case, the CP and Cauchy tests are compared with the Fisher-type test along the conventional contour suggested by Phillips and Sul (2001), which we denote by the PS test.

To implement the panel unit root test CP based on the OLS $t$-ratios, we need to adjust the given samples in all cross-sections so that they all have the same sum of squares of the lagged levels of the instrument, which is simply $\left(y_{i, t-1}\right)$ in this case. To this end, we fix the contour across all the cross-sections at the sum of squares of $\left(y_{i, t-1}\right)$ given by $c T^{2}$ with $c=3.5,2.5,1.5,0.5$, respectively for the sample sizes $T=25,50,100,200$. We are therefore fixing the sum of squares at the preset level $c T^{2}$ for all $N$ cross-sections in the panel samples generated from each of the 10,000 iterations we carry out. This is to enforce the same contour for all $N$ cross-sections in 10,000 realized panel samples in our simulations. The given data are then adjusted to be on the equi-squared-sum contour according to the given levels of sum of squares. ${ }^{5}$ For those cross-sections with the sum of squares greater

\footnotetext{
${ }^{5}$ In the simulations, the equi-squared-sum contour is defined by fixing the sum of squares of the lagged level $y_{i, t-1}$ after purging away the effects of short-run dynamics, i.e.,

$$
y_{i, t-1}^{p}=y_{i, t-1}-\left(\sum_{t=1}^{T} y_{i, t-1} x_{i t}^{\prime}\right)\left(\sum_{t=1}^{T} x_{i t} x_{i t}^{\prime}\right)^{-1} x_{i t}
$$
}


than the required level, some of the given observations are discarded, while for those units with deficient sum of squares, the bootstrapped data are added to extend the given sample until the target sum of squares is reached following the steps outlined in Section 4. Once all of the cross-sections reach the equi-squared-sum contour, the $t$-ratios based on the least squares estimators are computed for each individual units along the new contour. For the dependent panels, we first defactor the data ${ }^{6}$, and adjust the samples in the individual cross-sections to be on the equi-squared-sum contour as described above. We then compute the OLS $t$-tests along the new contour to construct our panel unit root tests.

For the implementation of the $\mathrm{CP}$ test to an actual panel data set, we may simply calculate the sum of squares for each cross-section, and fix the contour at the maximum of those individual sum of squares. We may then reach the contour by extending the samples using our bootstrap procedure for all individual units until their sum of squares reach the maximum. This is quite natural and clear. There is, however, one issue that needs to be addressed here. Unlike the simulations, we only have one realized panel sample when we work with a real data set. This means we are carrying out the bootstrap procedure only once for each cross-section. The generated bootstrap samples for the sample extensions will therefore be dependent on the random numbers drawn from the uniform distribution that we use to create the indexes for resampling. Consequently the resulting test will be affected by the randomness, producing different test results for different seeds used in the random number generator. The dependence of our procedure on the random numbers is mitigated in simulation exercises as we go through numerous iterations. When implemented to an actual data set, however, the randomness of our CP test will remain. To deal with such arbitrariness, we may try several seed numbers for the uniform random number generator and see if the test results are robust. Or, we may take a fixed set of uniform random numbers that I would provide, and use it as an input of our test procedure to ensure that we obtain the same result whenever the procedure is applied for the same data set. Then there will be no more arbitrariness in our testing procedure.

On the other hand, the implementation of the Cauchy panel test is strightforward, both in the simulations and for actual data, since there is no need for sample adjustments to be on the new contour. As mentioned earlier, the Cauchy $t$-ratios present a rare case where the equi-squared-sum and the conventional equi-sample-size contour coincide. Hence, we may just use the entire data given, as we normally do, to compute the Cauchy $t$-ratios for each cross-section, and combine them with the normalization $N^{-1 / 2}$ as in (28). For the dependent panels, defactor the data first to remove the cross-section dependency and then compute the individual Cauchy tests for all cross-sections to construct the Cauchy panel test as described above.

Table 1 presents for the independent case the performance of the averaged $t$-ratios based on the OLS and Cauchy estimators along the new equi-squared-sum contour, and the average $t$-test along the conventional contour with the mean and varaince modifications considered in Im, Pesaran and Shin (2003). We may summarize our findings here as follows. First, both the OLS and Cauchy based average $t$-ratio statistics along the new contour have very good

where $x_{i t}=\left(\triangle y_{i, t-1}, \ldots, \triangle y_{i, t-p+1}\right)^{\prime}$. Of course we may just use the sum of squares of the lagged level $y_{i, t-1}$ to define the contour, as suggested in the paper. They are asymptotically equivalent.

${ }^{6}$ We used the defactoring procedure suggested by Phillips and Sul (2001). 
finite sample sizes for all nominal test sizes and for all sample sizes considered here. Second, the Cauchy based average $t$-ratio statistic is more powerful than the OLS counterpart along the new contour, especially for the cases with smaller $N$ and $T$. The reason that the OLS based CP test turned out to be less powerful than the Cauchy test is that we are either discarding some of the given observations or relying on the bootstrapped data to be able to take the new equi-squared-sum contour. One might have well expected that the OLS based test would be more powerful, since the OLS estimator is more efficient than the Cauchy estimator. As our simulation results indicate, however, the relative efficiency of the OLS estimator over the Cauchy estimator is not large enough to pay off the cost of adjusting the sample for the OLS $t$-ratios to be on the new contour. The required data adjustment to be on the new contour can be costly in terms of power in panels with small cross-section and time series dimensions. However, the difference in the discriminatory powers between the two tests vanishes as both $N$ and $T$ increase. Third, the IPS test suffers from the size distortions which are increasing with $N$, especially when $T$ is small. See the case with $N=200$ and $T=25$, for instance. This indicates that the normalizations taken for the IPS test via the mean and variance modifications are indeed quite poor when $T$ is small even for the independent panels. Worse these errors in normalizations accumulate as $N$ increases, leading to the serious size distortions as we observe here.

Table 2 presents the simulation results for the dependent case. In general, our observations on the average $t$-ratios based on the OLS and Cauchy estimators for the independent case continue to apply for this case. Again, both CP and Cauchy tests along the new contour perform well in terms of the sizes, approximating very closely the nominal test sizes for all the sample sizes considered here. The Cauchy test again has higher discriminatory powers compared to the OLS based CP test in smaller panels. The powers of the both tests are in general smaller in the dependent panels compared to their powers in the independent panels. The Fisher-type test along the conventional contour by Phillips and Sul (2001) performs quite well in terms of the finite sample sizes. However, its discriminatory powers are extremely low, and worse it does not improve even when the sample size gets large.

Tables 3 and 4 report the finite sample performances of the maximum statistics for the independent and dependent cases, respectively. The CP and Cauchy maximum tests are respectively the maximums of the individual OLS and Cauchy $t$-ratios along the new contour. The IPS and PS tests are not considered here since they are not valid for testing the composite null hypothesis that we consider here. Overall the maximum tests have stable finite sample sizes and good power properties, although their performances are not as good as those of the average tests reported in Tables 1 and 2. Their finite sample performances, however, do improve as the sample size gets large. The $\mathrm{CP}$ maximum test in general has better sizes than the Cauchy maximum test, which tends to under-reject when $N$ is larger than $T$. The Cauchy maximum test, however, continues to be more powerful than the OLS counterpart, and the superiority of the Cauchy maximum test is more evident in the dependent case as can be seen from Table 4 . 


\section{Conclusion}

In this paper, we develop the new asymptotics for the unit root test based on the nonlinear IV $t$-ratio. It relies on the limit theories along the new contour given by the same sum of squared IV's, in contrast to the conventional contour given by the same sample size. In particular, we show in the paper that the asymptotics along the contour of the equi-squaredsum of IV's generally yield the normal asymptotics. Subsequently, we demonstrate that this finding may be exploited to invent tools and methodologies for the effective inferences in nonstationary panels. In the panel context, our theory implies that the individual tests behave asymptotically as normal when they are computed simply along the equi-squaredsum contour across the individual units. Consequently, we may use various functionals of those individual tests to do inference in nonstationary panels. Here we only concentrate on the panel unit root models. This is just for expositional simplicity, which was intended to deliver the main contents of the proposed methodology more clearly. Quite obviously, we may use essentially the same approach to develop the corresponding methods of inference that are applicable for cointegrated panels.

\section{Appendix: Mathematical Proofs}

Proof of Lemma 2.1 The limit theory under the null is derived in Phillips, Park and Chang (2004). For the limit theory under that local-to-unity alternative specified in (6), let

$$
W_{\delta T}=T^{-1 / 2} y_{[T r]}
$$

where $[x]$ denotes the largest integer not exceeding $x \geq 0$. It follows that

$$
W_{\delta T}(r) \rightarrow_{d} W_{\delta}(r)
$$

where $W_{\delta}$ is defined in $(7)$, uniformly in $D(\mathbb{R})$ as $T \rightarrow \infty$, which implies $H\left(W_{\delta T}\right) \rightarrow{ }_{d} H\left(W_{\delta}\right)$ uniformly in $D(\mathbb{R})$ as well. This is well known.

Under the local-to-unity alternative $\beta=-\delta / T$, we have

$$
\begin{aligned}
& R(T)= \frac{1}{\sigma}\left(\sum_{t=1}^{T} F\left(y_{t-1}\right)^{2}\right)^{-1 / 2}\left(-\frac{\delta}{T} \sum_{t=1}^{T} F\left(y_{t-1}\right) y_{t-1}+\sum_{t=1}^{T} F\left(y_{t-1}\right) \varepsilon_{t}\right) \\
&=-\frac{\delta}{\sigma}\left(\frac{1}{T \kappa(\sqrt{T})^{2}} \sum_{t=1}^{T} F\left(y_{t-1}\right)^{2}\right)^{-1 / 2} \frac{1}{T} \frac{1}{\sqrt{T} \kappa(\sqrt{T})} \sum_{t=1}^{T} F\left(y_{t-1}\right) y_{t-1} \\
&+\frac{1}{\sigma}\left(\frac{1}{T \kappa(\sqrt{T})^{2}} \sum_{t=1}^{T} F\left(y_{t-1}\right)^{2}\right)^{-1 / 2} \frac{1}{\sqrt{T} \kappa(\sqrt{T})} \sum_{t=1}^{T} F\left(y_{t-1}\right) \varepsilon_{t} \\
&=-\frac{\delta}{\sigma}\left(\int_{0}^{1} H\left(W_{\delta T}(r)\right)^{2} d r\right)^{-1 / 2} \int_{0}^{1} H\left(W_{\delta T}(r)\right) W_{\delta T}(r) d r \\
&+\frac{1}{\sigma}\left(\int_{0}^{1} H\left(W_{\delta T}(r)\right)^{2} d r\right)^{-1 / 2} \int_{0}^{1} H\left(W_{\delta T}(r)\right) d W_{T}(r)
\end{aligned}
$$




$$
\begin{aligned}
\rightarrow_{d}-\frac{\delta}{\sigma} & \left(\int_{0}^{1} H\left(W_{\delta}(r)\right)^{2} d r\right)^{-1 / 2} \int_{0}^{1} H\left(W_{\delta}(r)\right) W_{\delta}(r) d r \\
& +\frac{1}{\sigma}\left(\int_{0}^{1} H\left(W_{\delta}(r)\right)^{2} d r\right)^{-1 / 2} \int_{0}^{1} H\left(W_{\delta}(r)\right) d W(r)
\end{aligned}
$$

as $T \rightarrow \infty$, and this proves the stated result.

Proof of Theorem 2.2 Assume (2). Define $W_{T}(r)=T^{-1 / 2} y_{[T r]}$. It is well known that $W_{T} \rightarrow_{d} W$ in the space $D(\mathbb{R})$ of cadlag functions endowed with the supremum norm. Moreover, by extending the underlying probability space if necessary, we may always find from the same probability space a distributionally equivalent copy of $W_{T}$, say $W_{T}^{\prime}$, such that $W_{T}^{\prime} \rightarrow a . s W$ uniformly. Since we are interested only in the distributional results, we may assume $W_{T}$ and $W$ are defined in the same probability space and that $W_{T} \rightarrow a . s W$ uniformly. Such a construction is possible for instance by the Skorohod embedding. For more formal discussions on this, see Hall and Heyde (1980).

For any fixed constant $c>0$, we define $\tau_{T}(c)$ as

$$
\int_{0}^{\tau_{T}(c)} H\left(W_{T}(r)\right)^{2} d r=c
$$

and define a stopping time $\tau(c)$ to be such that

$$
\int_{0}^{\tau(c)} H(W(r))^{2} d r=c .
$$

Then we have

$$
\tau_{T}(c) \rightarrow a . s . \tau(c)
$$

as $T \rightarrow \infty$, since $H\left(W_{T}\right) \rightarrow a . s . H(W)$ uniformly. Note also that $\tau_{T}(c)=S_{T} / T+O\left(T^{-1}\right)$ a.s., from which we may further deduce that

$$
\frac{S_{T}}{T} \rightarrow a . s . \tau(c)
$$

as $T \rightarrow \infty$.

Under the null hypothesis of unit root $\beta=0$, we have

$$
\begin{aligned}
R\left(S_{T}\right) & =\frac{1}{\sigma}\left(\sum_{t=1}^{S_{T}} F\left(y_{t-1}\right)^{2}\right)^{-1 / 2} \sum_{t=1}^{S_{T}} F\left(y_{t-1}\right) \varepsilon_{t} \\
& =\frac{1}{\sigma}\left(\frac{1}{T \kappa(\sqrt{T})^{2}} \sum_{t=1}^{S_{T}} F\left(y_{t-1}\right)^{2}\right)^{-1 / 2} \frac{1}{\sqrt{T} \kappa(\sqrt{T})} \sum_{t=1}^{S_{T}} F\left(y_{t-1}\right) \varepsilon_{t} \\
& =\frac{1}{\sigma}\left(\int_{0}^{S_{T} / T} H\left(W_{T}(r)\right)^{2} d r\right)^{-1 / 2} \int_{0}^{S_{T} / T} H\left(W_{T}(r)\right) d W_{T}(r) \\
& =\left(\int_{0}^{\tau(c)} H(W(r))^{2} d r\right)^{-1 / 2} \frac{1}{\sigma} \int_{0}^{\tau(c)} H(W(r)) d W(r)+o(1) \text { a.s. }
\end{aligned}
$$


as $T \rightarrow \infty$, since $H\left(W_{T}\right) \rightarrow_{a . s .} H(W)$ uniformly and $S_{T} / T \rightarrow_{a . s .} \tau(c)$ as $T \rightarrow \infty$. However, the process $U$ defined by

$$
U(s)=\frac{1}{\sigma} \int_{0}^{\tau(s)} H(W(r)) d W(r)
$$

is the DDS Brownian motion of the martingale $M$

$$
M(s)=\frac{1}{\sigma} \int_{0}^{s} H(W(r)) d W(r)
$$

and therefore,

$$
\left(\int_{0}^{\tau(c)} H(W(r))^{2} d r\right)^{-1 / 2} \frac{1}{\sigma} \int_{0}^{\tau(c)} H(W(r)) d W(r)=c^{-1 / 2} U(c) \sim \mathbf{N}(0,1)
$$

for any given $c>0$. The reader is referred to, e.g., Revuz and Yor (1994) for the DDS Brownian motion. The stated result now follows readily from (31) and (32).

Next, assume (6). If we define $\tau_{\delta}(c)$ by

$$
\int_{0}^{\tau_{\delta}(c)} H\left(W_{\delta}(r)\right)^{2} d r=c
$$

for a given fixed $c>0$, then $S_{T} / T \rightarrow_{a . s .} \tau_{\delta}(c)$ exactly as in the proof for the null distribution given above.

Under the alternative of local-to-unity $\beta=-\delta / T$, we have

$$
\begin{aligned}
& R\left(S_{T}\right)= \frac{1}{\sigma}\left(\sum_{t=1}^{S_{T}} F\left(y_{t-1}\right)^{2}\right)^{-1 / 2}\left(-\frac{\delta}{T} \sum_{t=1}^{S_{T}} F\left(y_{t-1}\right) y_{t-1}+\sum_{t=1}^{S_{T}} F\left(y_{t-1}\right) \varepsilon_{t}\right) \\
&=-\frac{\delta}{\sigma}\left(\sum_{t=1}^{S_{T}} F\left(y_{t-1}\right)^{2}\right)^{-1 / 2} \frac{1}{T} \sum_{t=1}^{S_{T}} F\left(y_{t-1}\right) y_{t-1} \\
&+\frac{1}{\sigma}\left(\sum_{t=1}^{S_{T}} F\left(y_{t-1}\right)^{2}\right)^{-1 / 2} \sum_{t=1}^{S_{T}} F\left(y_{t-1}\right) \varepsilon_{t} \\
&=-\frac{\delta}{\sigma}\left(\int_{0}^{S_{T} / T} H\left(W_{\delta T}(r)\right)^{2} d r\right)^{-1 / 2} \int_{0}^{S_{T} / T} H\left(W_{\delta T}(r)\right) W_{\delta T}(r) d r \\
& \quad+\frac{1}{\sigma}\left(\int_{0}^{S_{T} / T} H\left(W_{\delta T}(r)\right)^{2} d r\right)^{-1 / 2} \int_{0}^{S_{T} / T} H\left(W_{\delta T}(r)\right) d W_{T}(r) \\
&=-\frac{\delta}{\sigma}\left(\int_{0}^{\tau(c)} H\left(W_{\delta}(r)\right)^{2} d r\right)^{-1 / 2} \int_{0}^{\tau(c)} H\left(W_{\delta}(r)\right) W_{\delta}(r) d r \\
& \quad+\quad\left(\int_{0}^{\tau(c)} H\left(W_{\delta}(r)\right)^{2} d r\right)^{-1 / 2} \frac{1}{\sigma} \int_{0}^{\tau(c)} H\left(W_{\delta}(r)\right) d W(r)+o(1) \text { a.s. }
\end{aligned}
$$


as $T \rightarrow \infty$. We now consider the DDS Brownian motion

$$
U_{\delta}(s)=\frac{1}{\sigma} \int_{0}^{\tau_{\delta}(s)} H\left(W_{\delta}(r)\right) d W(r)
$$

of the martingale

$$
M_{\delta}(s)=\frac{1}{\sigma} \int_{0}^{s} H\left(W_{\delta}(r)\right) d W(r),
$$

from which the stated result follows immediately, due to (33) and (34).

Proof of Corollary 3.1 Denote by $W$ the limit Brownian motion appearing in the invariance principle introduced in (3). If we let

$$
W_{T}^{*}(r)=\frac{1}{\sqrt{T}} \sum_{t=1}^{[T r]} \varepsilon_{t}^{*},
$$

for $r \geq 0$, then we have

$$
W_{T}^{*} \rightarrow d^{*} W^{*} \text { a.s. }
$$

as $T \rightarrow \infty$. The reader is referred to Park (2003) for more details on the bootstrap invariance principle. Since the limit distribution is not dependent upon the realization of the sample, we may deduce that $W^{*}$ is independent of $W$.

The first part on the null limit distribution follows exactly as in the proof for the first part of Theorem 2.2. We let $W_{T}^{+}(r)=T^{-1 / 2} y_{[T r]}^{+}$. As before we have $W_{T}^{+} \rightarrow_{a . s .} W^{+}$uniformly, where the process $W^{+}$is defined in (10), and this in turn implies $H\left(W_{T}^{+}\right) \rightarrow_{a . s .} H\left(W^{+}\right)$ uniformly as well. We also define $\tau_{T}(c)$ for a fixed constant $c$ as

$$
\int_{0}^{\tau_{T}(c)} H\left(W_{T}^{+}(r)\right)^{2} d r=c
$$

and define a stopping time $\tau(c)$ as

$$
\int_{0}^{\tau(c)} H\left(W^{+}(r)\right)^{2} d r=c .
$$

Similarly as in the proof of Theorem 2.2, it follows that $\tau_{T}(c) \rightarrow$ a.s. $\tau(c)$, which implies $S_{T}^{+} / T \rightarrow a . s . \tau(c)$, since $\tau_{T}(c)=S_{T}^{+} / T+O\left(T^{-1}\right)$. The rest of the proof now follows exactly as in the proof of the first part of Theorem 2.2.

For the second part, the extended samples behave like near unit root processes up to $T$, and then like the processes with exact unit root thereafter. For more detailed discussion on the bootstrap theory for the near unit root processes, see Park (2004). When $S_{T}^{+} \leq T$, $S_{T}^{+}=S_{T}$ and the limit theory under the local-to-unity alternative follows exactly as in the proof for the second part of Theorem 2.2. Hence, we consider the case $S_{T}^{+}>T$. We let 
$W_{\delta T}^{+}(r)=T^{-1 / 2} y_{[T r]}^{+}$. We then have $W_{\delta T}^{+} \rightarrow a . s . W_{\delta}^{+}$and $H\left(W_{T}^{+}\right) \rightarrow_{a . s .} H\left(W_{\delta}^{+}\right)$uniformly, where $W_{\delta}^{+}$is defined in (11). Here we define $\tau_{T}(c)$ for a fixed constant $c$ as

$$
\int_{0}^{\tau_{T}(c)} H\left(W_{\delta T}^{+}(r)\right)^{2} d r=c,
$$

and define a stopping time $\tau(c)$ as

$$
\int_{0}^{\tau(c)} H\left(W_{\delta}^{+}(r)\right)^{2} d r=c
$$

We also have $S_{T}^{+} / T \rightarrow_{a . s .} \tau(c)$, since $\tau_{T}(c) \rightarrow_{a . s .} \tau(c)$ and $\tau_{T}(c)=S_{T}^{+} / T+O\left(T^{-1}\right)$ hold here.

Notice that

$$
\triangle y_{t}^{+}=\left\{\begin{array}{ll}
\triangle y_{t}=-\frac{\delta}{T} y_{t-1}+\varepsilon_{t} & \text { if } t \leq T \\
\triangle y_{t}^{*}=\varepsilon_{t}^{*} & \text { if } t \geq T+1
\end{array} .\right.
$$

Then we have

$$
\sum_{t=1}^{S_{T}^{+}} F\left(y_{t-1}^{+}\right) \triangle y_{t}^{+}=-\frac{\delta}{T} \sum_{t=1}^{T} F\left(y_{t-1}\right) y_{t-1}+\sum_{t=1}^{T} F\left(y_{t-1}\right) \varepsilon_{t}+\sum_{t=T+1}^{S_{T}^{+}} F\left(y_{t-1}^{*}\right) \varepsilon_{t}^{*}
$$

and

$$
\begin{aligned}
& \frac{1}{\sqrt{T} \kappa(\sqrt{T})} \sum_{t=1}^{S_{T}^{+}} F\left(y_{t-1}^{+}\right) \Delta y_{t}^{+} \\
& =-\frac{\delta}{T} \frac{1}{\sqrt{T} \kappa(\sqrt{T})} \sum_{t=1}^{T} F\left(y_{t-1}\right) y_{t-1}+\frac{1}{\sqrt{T} \kappa(\sqrt{T})} \sum_{t=1}^{T} F\left(y_{t-1}\right) \varepsilon_{t} \\
& \quad+\frac{1}{\sqrt{T} \kappa(\sqrt{T})} \sum_{t=T+1}^{S_{T}^{+}} F\left(y_{t-1}^{*}\right) \varepsilon_{t}^{*} \\
& =-\delta \int_{0}^{1} H\left(W_{\delta T}(r)\right) W_{\delta T}(r) d r+\int_{0}^{1} H\left(W_{\delta T}(r)\right) d W_{T}(r)+\int_{1}^{S_{T}^{+} / T} H\left(W_{T}^{*}(r)\right) d W_{T}^{*}(r) \\
& =-\delta \int_{0}^{1} H\left(W_{\delta}(r)\right) W_{\delta}(r) d r+\int_{0}^{1} H\left(W_{\delta}(r)\right) d W+\int_{1}^{\tau(c)} H\left(W^{*}(r)\right) d W^{*}(r)+o(1) \quad \text { a.s. } \\
& =-\delta \int_{0}^{1} H\left(W_{\delta}(r)\right) W_{\delta}(r) d r+\int_{0}^{\tau(c)} H\left(W_{\delta}^{+}(r)\right) d W^{+}(r)
\end{aligned}
$$

due to the bootstrap invariance principle given in (35) and the definitions of the extended Brownian motions $W^{+}$and $W_{\delta}^{+}$provided in (10) and (11). 
We may then easily deduce that under $\beta=-\delta / T$ and $S_{T}^{+}>T$,

$$
\begin{aligned}
R\left(S_{T}^{+}\right)= & \frac{1}{\sigma}\left(\sum_{t=1}^{S_{T}^{+}} F\left(y_{t-1}^{+}\right)^{2}\right)^{-1 / 2} \sum_{t=1}^{S_{T}^{+}} F\left(y_{t-1}^{+}\right) \triangle y_{t}^{+} \\
= & -\frac{\delta}{\sigma}\left(\int_{0}^{\tau(c)} H\left(W_{\delta}^{+}(r)\right)^{2} d r\right)^{-1 / 2} \int_{0}^{1} H\left(W_{\delta}(r)\right) W_{\delta}(r) d r \\
& +\frac{1}{\sigma}\left(\int_{0}^{\tau(c)} H\left(W_{\delta}^{+}(r)\right)^{2} d r\right)^{-1 / 2} \int_{0}^{\tau(c)} H\left(W_{\delta}^{+}(r)\right) d W^{+}(r)+o(1) \text { a.s. }
\end{aligned}
$$

as $T \rightarrow \infty$. The stated result is now immediate.

Proof of Corollary 4.1 The nonlinear IV $t$-ratio $\tilde{R}(T)$ for testing $\beta=0$ in the regression (14) can be written as

$$
\tilde{R}(T)=(1 / \sigma) Q_{T}^{-1 / 2} P_{T}
$$

where

$$
\begin{aligned}
P_{T} & =\sum_{t=1}^{T} F\left(\tilde{y}_{t-1}\right) \Delta y_{t}-\left(\sum_{t=1}^{T} F\left(\tilde{y}_{t-1}\right) x_{t}^{\prime}\right)\left(\sum_{t=1}^{T} x_{t} x_{t}^{\prime}\right)^{-1}\left(\sum_{t=1}^{T} x_{t} \Delta y_{t}\right) \\
Q_{T} & =\sum_{t=1}^{T} F\left(\tilde{y}_{t-1}\right)^{2}-\left(\sum_{t=1}^{T} F\left(\tilde{y}_{t-1}\right) x_{t}^{\prime}\right)\left(\sum_{t=1}^{T} x_{t} x_{t}^{\prime}\right)^{-1}\left(\sum_{t=1}^{T} x_{t} F\left(\tilde{y}_{t-1}\right)\right)
\end{aligned}
$$

and $x_{t}=\left(\triangle y_{t-1}, \ldots, \triangle y_{t-p+1}\right)^{\prime}$.

Under the demeaning scheme (15), $\triangle y_{t}=\triangle y_{t}^{\circ}$ and $\tilde{y}_{t-1}=y_{t-1}^{\circ}$, and thus the regression (14) becomes exactly as the regression given in (13). Let $x_{t}^{\circ}=\left(\triangle y_{t-1}^{\circ}, \ldots, \triangle y_{t-p+1}^{\circ}\right)^{\prime}$, and define $P_{1 T}^{\circ}$ and $Q_{1 T}^{\circ}$ exactly the same as $P_{T}$ and $Q_{T}$, but with $\triangle y_{t}^{\circ}, y_{t-1}^{\circ}$ and $x_{t}^{\circ}$ in the places of $\triangle y_{t}, \tilde{y}_{t-1}$ and $x_{t}$. Then under the demeaning scheme (15), we have

$$
P_{T}=P_{T}^{\circ}, \quad Q_{T}=Q_{T}^{\circ}
$$

and the IV $t$-ratio $\tilde{R}(T)$ can now be written equivalently as $\tilde{R}(T)=(1 / \sigma) Q_{T}^{\circ-1 / 2} P_{T}^{\circ}$.

To derive the limit theory, let

$$
u_{t}=\sum_{k=1}^{p-1} \alpha_{k} \triangle y_{t-k}^{\circ}+\varepsilon_{t}
$$

and suppose that $\alpha(z)=1-\sum_{k=1}^{p-1} \alpha_{k} z^{k}$ and $\left(\varepsilon_{t}\right)$ satisty the standard conditions routinely imposed in unit root models, such as those in Chang and Park (2002). Then the following invariance principle holds:

$$
\frac{1}{\sqrt{T}} \sum_{t=1}^{[T r]} u_{t} \rightarrow_{d} V(r)
$$


where the limit BM $V$ is defined in (18). Assume $y_{1}^{\circ}=0$ for simplicity. Under the null of unit root $\beta=0$, we have $\triangle y_{t}^{\circ}=u_{t}$, which implies

$$
T^{-1 / 2} y_{[T r]}^{\circ}=T^{-1 / 2} \sum_{t=1}^{[T r]} u_{t} \rightarrow_{d} V(r)
$$

as $T \rightarrow \infty$, and

$$
\begin{aligned}
\frac{1}{\kappa(\sqrt{T}) \sqrt{T}} \sum_{t=1}^{T} F\left(y_{t-1}^{\circ}\right) \Delta y_{t}^{\circ} & =\frac{1}{\kappa(\sqrt{T}) \sqrt{T}} \sum_{t=1}^{T} F\left(y_{t-1}^{\circ}\right) u_{t} \rightarrow_{d} \int_{0}^{1} H(V(r)) d V(r) \\
\frac{1}{\kappa^{2}(\sqrt{T}) T} \sum_{t=1}^{T} F\left(y_{t-1}^{\circ}\right)^{2} & \rightarrow_{d} \int_{0}^{1} H(V(r))^{2} d r
\end{aligned}
$$

Also under the null, $\triangle y_{t-k}^{\circ}=u_{t-k}$, implying $\sum_{t=1}^{T} F\left(y_{t-1}^{\circ}\right) \triangle y_{t-k}^{\circ}=O_{p}\left(\kappa(\sqrt{T}) T^{1 / 2}\right)$ and $\sum_{t=1}^{T} \triangle y_{t-k}^{\circ} \triangle y_{t}^{\circ}=\sum_{t=1}^{T} u_{t-k} u_{t}=O_{p}(\sqrt{T})$, for each $k=1, \ldots, p-1$, and $\sum_{t=1}^{T} \triangle y_{t-k}^{\circ} \triangle y_{t-\ell}^{\circ}=$ $\sum_{t=1}^{T} u_{t-k} u_{t-\ell}=O_{p}(T)$, for all $k, \ell=1, \ldots, p-1$. We may now deduce that

$$
\sum_{t=1}^{T} F\left(y_{t-1}^{\circ}\right) x_{t}^{\circ \prime}=O_{p}(\kappa(\sqrt{T}) \sqrt{T}), \quad \sum_{t=1}^{T} x_{t}^{\circ} x_{t}^{\circ \prime}=O_{p}(T), \quad \sum_{t=1}^{T} x_{t}^{\circ} \triangle y_{t}^{\circ}=O_{p}(\sqrt{T})
$$

which in turn implies

$$
\begin{aligned}
\frac{1}{\kappa(\sqrt{T}) \sqrt{T}} P_{T}^{\circ} & =\frac{1}{\kappa(\sqrt{T}) \sqrt{T}} \sum_{t=1}^{T} F\left(y_{t-1}^{\circ}\right) \triangle y_{t}^{\circ}+O_{p}\left(T^{-1 / 2}\right) \\
\frac{1}{\kappa(\sqrt{T})^{2} T} Q_{T}^{\circ} & =\frac{1}{\kappa(\sqrt{T})^{2} T} \sum_{t=1}^{T} F\left(y_{t-1}^{\circ}\right)^{2}+O_{p}\left(T^{-1}\right)
\end{aligned}
$$

The null limit distributions of $\tilde{R}(T)$ and $R\left(\tilde{S}_{T}\right)$ can now be derived as in Lemma 2.1 and Theorem 2.2 using the results given in (38) and (39) due to (36) and (37).

Under the local-to-unity alternative $\beta=-\delta / T$,

$$
T^{-1 / 2} y_{[T r]}^{\circ} \rightarrow_{d} V_{\delta}(r)
$$

the Ornstein-Ulenbeck process defined in (19), and

$$
\triangle y_{t}^{\circ}=-(\delta / T) y_{t-1}^{\circ}+u_{t}
$$

Then it follows that

$$
\begin{aligned}
& \frac{1}{\kappa(\sqrt{T}) \sqrt{T}} \sum_{t=1}^{T} F\left(y_{t-1}^{\circ}\right) \triangle y_{t}^{\circ} \\
& =-\frac{\delta}{\kappa(\sqrt{T}) T^{3 / 2}} \sum_{t=1}^{T} F\left(y_{t-1}^{\circ}\right) y_{t-1}^{\circ}+\frac{1}{\kappa(\sqrt{T}) \sqrt{T}} \sum_{t=1}^{T} F\left(y_{t-1}^{\circ}\right) u_{t} \\
& \rightarrow_{d} \quad-\delta \int_{0}^{1} H\left(V_{\delta}(r)\right) V_{\delta}(r) d r+\int_{0}^{1} H\left(V_{\delta}(r)\right) d V(r)
\end{aligned}
$$


and

$$
\frac{1}{\kappa^{2}(\sqrt{T}) T} \sum_{t=1}^{T} F\left(y_{t-1}^{\circ}\right)^{2} \rightarrow d \int_{0}^{1} H\left(V_{\delta}(r)\right)^{2} d r
$$

With $\beta=-\delta / T$, we have

$$
\begin{aligned}
\triangle y_{t-k}^{\circ} \triangle y_{t}^{\circ} & =-\frac{\delta^{2}}{T^{2}} y_{t-k-1}^{\circ} y_{t-1}^{\circ}-\frac{\delta}{T} y_{t-k-1}^{\circ} u_{t}-\frac{\delta}{T} y_{t-1}^{\circ} u_{t-k}+u_{t-k} u_{t} \\
\triangle y_{t-k}^{\circ} \triangle y_{t-\ell}^{\circ} & =-\frac{\delta^{2}}{T^{2}} y_{t-k-1}^{\circ} y_{t-\ell-1}^{\circ}-\frac{\delta}{T} y_{t-k-1}^{\circ} u_{t-\ell}-\frac{\delta}{T} y_{t-\ell-1}^{\circ} u_{t-k}+u_{t-k} u_{t-\ell}
\end{aligned}
$$

for $k, \ell=1, \ldots, p-1$, implying

$$
\begin{gathered}
\sum_{t=1}^{T} \triangle y_{t-k}^{\circ} \triangle y_{t}^{\circ}=O_{p}(1)+O_{p}(1)+O_{p}(\sqrt{T})+O_{p}(\sqrt{T})=O_{p}(\sqrt{T}) \\
\sum_{t=1}^{T} \triangle y_{t-k}^{\circ} \triangle y_{t-\ell}^{\circ}=O_{p}(1)+O_{p}(\sqrt{T})+O_{p}(\sqrt{T})+O_{p}(T)=O_{p}(T)
\end{gathered}
$$

Hence the results in (40) on the stochastic orders of the component sample moments in $P_{T}^{\circ}$ and $Q_{T}^{\circ}$ also hold under the local-to-unity alternative. The stated limit theories for the tests $\tilde{R}(T)$ and $R\left(\tilde{S}_{T}\right)$ under $\beta=-\delta / T$ then follow just as in Lemma 2.1 and Theorem 2.2 due to (36), (37), (41) and (42).

Proof of Corollary 4.2 With the demeaning scheme (16),

$$
\tilde{y}_{t}=\tilde{y}_{t}^{\circ}=y_{t}^{\circ}-\frac{1}{t} \sum_{k=1}^{t} y_{k}^{\circ}
$$

and the regression (14) can be rewritten as

$$
\triangle y_{t}^{\circ}=\beta \tilde{y}_{t-1}^{\circ}+\sum_{k=1}^{p-1} \alpha_{k} \Delta y_{t-k}^{\circ}+\varepsilon_{t},
$$

since $\Delta y_{t-s}=\triangle y_{t-s}^{\circ}$, for $s=0,1, \ldots, p-1$, and $\tilde{y}_{t-1}=\tilde{y}_{t-1}^{\circ}$. As in the proof of Corollary 4.1, we may also define $P_{T}^{\circ}$ and $Q_{T}^{\circ}$ analogously as $P_{T}$ and $Q_{T}$, but now with $\tilde{y}_{t-1}^{\circ}$ in the place of $\tilde{y}_{t-1}$. The IV $t$-ratio from the regression (14) under the demeaning scheme (16) can be also written as $\tilde{R}(T)=(1 / \sigma) Q_{T}^{\circ-1 / 2} P_{T}^{\circ}$.

To derive the limit theories, note that

$$
T^{-1 / 2} \tilde{y}_{[T r]}^{\circ} \rightarrow d \begin{cases}V(r)-\frac{1}{r} \int_{0}^{r} V(s) d s=\tilde{V}(r), & \text { when } \beta=0 \\ V_{\delta}(r)-\frac{1}{r} \int_{0}^{r} V_{\delta}(s) d s=\tilde{V}_{\delta}(r), & \text { when } \beta=-\delta / T\end{cases}
$$


as $T \rightarrow \infty$, using the notations $\tilde{V}$ and $\tilde{V}_{\delta}$ introduced in (20) and (21). Similarly as before, we have under the null $\beta=0$,

$$
\frac{1}{\kappa(\sqrt{T}) \sqrt{T}} \sum_{t=1}^{T} F\left(\tilde{y}_{t-1}^{\circ}\right) \triangle y_{t}^{\circ}=\frac{1}{\kappa(\sqrt{T}) \sqrt{T}} \sum_{t=1}^{T} F\left(\tilde{y}_{t-1}^{\circ}\right) u_{t} \rightarrow_{d} \int_{0}^{1} H\left(\tilde{V}_{\delta}(r)\right) d V(r)
$$

and under the local-to-unity alternative $\beta=-\delta / T$,

$$
\begin{aligned}
& \frac{1}{\kappa(\sqrt{T}) \sqrt{T}} \sum_{t=1}^{T} F\left(\tilde{y}_{t-1}^{\circ}\right) \triangle y_{t}^{\circ} \\
& =-\frac{\delta}{\kappa(\sqrt{T}) T^{3 / 2}} \sum_{t=1}^{T} F\left(\tilde{y}_{t-1}^{\circ}\right) y_{t-1}^{\circ}+\frac{1}{\kappa(\sqrt{T}) \sqrt{T}} \sum_{t=1}^{T} F\left(\tilde{y}_{t-1}^{\circ}\right) u_{t} \\
& \rightarrow_{d} \quad-\delta \int_{0}^{1} H\left(\tilde{V}_{\delta}(r)\right) V_{\delta}(r) d r+\int_{0}^{1} H\left(\tilde{V}_{\delta}(r)\right) d V(r)
\end{aligned}
$$

Finally,

$$
\sum_{t=1}^{T} F\left(\tilde{y}_{t-1}^{\circ}\right) x_{t}^{\circ}=O_{p}(\kappa(\sqrt{T}) \sqrt{T})
$$

since $\sum_{t=1}^{T} F\left(\tilde{y}_{t-1}^{\circ}\right) \triangle y_{t-k}^{\circ}=O_{p}(\kappa(\sqrt{T}) \sqrt{T})$, for all $k=1, \ldots, p-1$, both under the null and the alternative. The rest of the proof is essentially identical to that of Corollary 4.1, given the results in Lemma 2.1 and in equations (43) and (44) above.

Proof of Corollary 4.3 The proof is immediate from our earlier results in Corollary 3.1 and the proofs of Corollaries 4.1 and 4.2 .

Proof of Theorem 5.1 The proof follows directly from our earlier results in Corollaries 4.2 and 4.3 , and the details are omitted.

\section{References}

Bai, J. and S. Ng (2004). "A PANIC attack on unit roots and cointegration," Econometrica $72,1127-1177$.

Baltagi, B.H. and C. Kao (2001). "Nonstationary panels, cointegration in panels and dynamic panels: A survey," mimeographed, Department of Economics, Texas A\& M University.

Banerjee, A. (1999). "Panel data unit roots and cointegration: An overview," Oxford Bulletin of Economics 83 Statistics 61: 607-629. 
Basawa, I.V., Mallik, A.K., McCormick, W.P., Reeves, J.H., Taylor, R.L. (1991). "Bootstrapping unstable first-order autoregressive processes," Annals of Statistics 19: 10981101.

Chang, Y. (2002). "Nonlinear IV unit root tests in panels with cross-sectional dependency," Journal of Econometrics, 110, 261-292.

Chang, Y. (2004). "Bootstrap unit root tests in panels with cross-sectional dependency," Journal of Econometrics 120: 263-293.

Chang, Y. and J.Y. Park (2002). "On the asymptotics of ADF tests for unit roots," Econometric Reviews 21: 431-447.

Chang, Y. and J.Y. Park (2003). "A sieve bootstrap for the test of a unit root," Journal of Time Series Analysis 24 (4), 379-400.

Chang, Y. and J.Y. Park (2004). "Taking a new contour: A novel view on unit root test," mimeographed, Department of Economics, Rice University.

Chang, Y. and W. Song (2002). "Panel unit root tests in the presence of cross-sectional dependency and heterogeneity," mimeographed, Department of Economics, Rice University.

Choi, I. (2001a). "Unit root tests for panel data," Journal of International Money and Finace 20: 219-247.

Choi, I. (2001b). "Unit root tests for cross-sectionally correlated panels," mimeographed, Kukmin University.

Dickey, D. A. and W. A. Fuller (1979). "Distribution of estimators for autoregressive time series with a unit root," Journal of the American Statistical Association 74, 427-431.

Dickey, D.A. and W.A. Fuller (1981). "Likelihood ratio statistics for autoregressive time series with a unit root," Econometrica 49, 1057-1072.

Fuller, W.A. (1996). Introduction to Statistical Time Series, 2nd ed. Wiley: New York.

Im, K., H. Pesaran and Y. Shin (2003). "Testing for unit roots in heterogeneous panels," Journal of Econometrics 115, pp.53-74.

Kao, C. (1999). "Spurious regression and residual-based tests for cointegration in panel data," Journal of Econometrics 90: 1-44.

Levin, A., C. Lin and C.J. Chu (2002). "Unit root tests in panel data: Asymptotic and finite-sample properties," Journal of Econometrics 108, 1-24.

Maddala, G.S. and S. Wu (1999). "A comparative study of unit root tests with panel data and a new simple test: Evidence from simulations and bootstrap," Oxford Bulletin of Economics and Statistics 61, 631-. 
Moon, H.R. and B. Perron, (2001). "Testing for a unit root in panels with dynamic factors," forthcoming in Journal of Econometrics.

Park, J.Y. (2003). "Bootstrap unit root tests," Econometrica 71: 1845-1895.

Park, J.Y. (2004). "Strong approximations for nonlinear transformations of integrated time series", mimeographed, Department of Economics, Rice University.

Park, J.Y. and P.C.B. Phillips (1999). "Asymptotics for nonlinear transformations of integrated time series," Econometric Theory, 15: 269-298.

Pedroni, P. and J.P. Urbain (2004), The Econometrics of Nonstationary Panels, forthcoming, Oxford University Press, Advanced Texts in Econometrics.

Pesaran, M.H. (2003). "A simple panel unit root test in the presence of cross section dependence," mimeographed, Department of Economics, Cambridge University.

Phillips, P.C.B. (1987). "Time series regression with a unit root," Econometrica 55, 277301.

Phillips, P.C.B. and H.R. Moon (1999). "Linear regression limit theory for nonstationary panel data," Econometrica 67: 1057-1111.

Phillips, P.C.B. and H.R. Moon (2000). "Nonstationary Panel Data Analysis: An Overview of Some Recent Developments," Econometric Reviews 19, 263-286.

Phillips, P.C.B., J.Y. Park and Y. Chang (2004). "Nonlinear instrumental variable estimation of an autoregression," Journal of Econometrics 118, 219-246.

Phillips, P.C.B. and D. Sul (2001). "Dynamic panel estimation and homogeneity testing under cross section dependence," mimeographed, University of Auckland.

Quah, D. (1994). Exploiting cross-section variations for unit root inference in dynamic data, Economics Letters, 44, 9-19.

Said, S.E. and D.A. Dickey (1984). "Testing for unit roots in autoregressive-moving average models of unknown order," Biometrika 71, 599-608.

So, B.S. and D.W. Shin (1999a). "Recursive mean adjustment in time-series inferences," Statistics and Probability Letters, 43, 65-73.

So, B.S. and D.W. Shin (1999b). "Cauchy estimators for autoregressive processes with applications to unit root tests and confidence intervals," Econometric Theory, 15, 165-176.

Stock, J.H. (1994). "Unit roots and structural breaks," In R.F. Engle and D. McFadden, eds., Handbook of Econometrics, Vol. 4, 2739-2841, Elsevier: Amsterdam.

Revuz, D. and M. Yor (1994). Continuous Martingale and Brownian Motion, 2nd ed. Springer-Verlag: New York. 
Table 1: Average t-Tests Along the Conventional and New Contours: Independent Case

\begin{tabular}{|c|c|c|c|c|c|c|c|c|c|c|c|}
\hline \multicolumn{3}{|c|}{ Constant only } & \multicolumn{3}{|c|}{ Sizes } & \multicolumn{3}{|c|}{ Rejection Probabilities } & \multicolumn{3}{|c|}{ Size-Adjusted Powers } \\
\hline$T$ & $N$ & Tests & $1 \%$ & $5 \%$ & $10 \%$ & $1 \%$ & $5 \%$ & $10 \%$ & $1 \%$ & $5 \%$ & $10 \%$ \\
\hline \multirow[t]{12}{*}{25} & \multirow[t]{3}{*}{10} & IPS & 0.025 & 0.097 & 0.165 & 0.052 & 0.168 & 0.271 & 0.022 & 0.098 & 0.174 \\
\hline & & $\mathrm{CP}$ & 0.010 & 0.056 & 0.106 & 0.032 & 0.129 & 0.219 & 0.031 & 0.117 & 0.208 \\
\hline & & Cauchy & 0.014 & 0.054 & 0.106 & 0.076 & 0.230 & 0.359 & 0.058 & 0.214 & 0.346 \\
\hline & \multirow[t]{3}{*}{50} & IPS & 0.046 & 0.143 & 0.232 & 0.169 & 0.391 & 0.529 & 0.054 & 0.182 & 0.305 \\
\hline & & $\mathrm{CP}$ & 0.013 & 0.064 & 0.119 & 0.089 & 0.259 & 0.394 & 0.074 & 0.227 & 0.352 \\
\hline & & Cauchy & 0.015 & 0.060 & 0.114 & 0.275 & 0.533 & 0.677 & 0.207 & 0.500 & 0.646 \\
\hline & \multirow[t]{3}{*}{100} & IPS & 0.069 & 0.194 & 0.296 & 0.345 & 0.601 & 0.729 & 0.090 & 0.274 & 0.435 \\
\hline & & $\mathrm{CP}$ & 0.015 & 0.067 & 0.128 & 0.195 & 0.438 & 0.579 & 0.146 & 0.375 & 0.525 \\
\hline & & Cauchy & 0.014 & 0.057 & 0.111 & 0.566 & 0.807 & 0.895 & 0.516 & 0.790 & 0.885 \\
\hline & \multirow[t]{3}{*}{200} & IPS & 0.111 & 0.272 & 0.387 & 0.652 & 0.851 & 0.917 & 0.225 & 0.474 & 0.624 \\
\hline & & $\mathrm{CP}$ & 0.017 & 0.071 & 0.141 & 0.403 & 0.673 & 0.793 & 0.312 & 0.596 & 0.735 \\
\hline & & Cauchy & 0.012 & 0.052 & 0.105 & 0.884 & 0.969 & 0.987 & 0.860 & 0.968 & 0.987 \\
\hline \multirow[t]{12}{*}{50} & \multirow[t]{3}{*}{10} & IPS & 0.014 & 0.066 & 0.124 & 0.040 & 0.150 & 0.255 & 0.030 & 0.115 & 0.216 \\
\hline & & $\mathrm{CP}$ & 0.010 & 0.049 & 0.102 & 0.042 & 0.157 & 0.263 & 0.042 & 0.158 & 0.258 \\
\hline & & Cauchy & 0.013 & 0.053 & 0.103 & 0.107 & 0.306 & 0.465 & 0.088 & 0.295 & 0.458 \\
\hline & \multirow[t]{3}{*}{50} & IPS & 0.021 & 0.081 & 0.152 & 0.182 & 0.423 & 0.571 & 0.110 & 0.325 & 0.461 \\
\hline & & $\mathrm{CP}$ & 0.012 & 0.058 & 0.112 & 0.186 & 0.441 & 0.590 & 0.169 & 0.415 & 0.565 \\
\hline & & Cauchy & 0.011 & 0.054 & 0.101 & 0.634 & 0.863 & 0.931 & 0.620 & 0.850 & 0.931 \\
\hline & \multirow[t]{3}{*}{100} & IPS & 0.026 & 0.105 & 0.184 & 0.447 & 0.724 & 0.839 & 0.289 & 0.579 & 0.714 \\
\hline & & $\mathrm{CP}$ & 0.012 & 0.060 & 0.119 & 0.489 & 0.745 & 0.851 & 0.449 & 0.717 & 0.821 \\
\hline & & Cauchy & 0.012 & 0.053 & 0.101 & 0.974 & 0.997 & 0.999 & 0.970 & 0.997 & 0.999 \\
\hline & \multirow[t]{3}{*}{200} & IPS & 0.040 & 0.124 & 0.208 & 0.811 & 0.944 & 0.977 & 0.594 & 0.847 & 0.926 \\
\hline & & $\mathrm{CP}$ & 0.015 & 0.066 & 0.125 & 0.838 & 0.954 & 0.981 & 0.813 & 0.937 & 0.971 \\
\hline & & Cauchy & 0.011 & 0.050 & 0.097 & 1.000 & 1.000 & 1.000 & 1.000 & 1.000 & 1.000 \\
\hline \multirow[t]{12}{*}{100} & \multirow[t]{3}{*}{10} & IPS & 0.012 & 0.058 & 0.110 & 0.110 & 0.332 & 0.496 & 0.100 & 0.299 & 0.471 \\
\hline & & $\mathrm{CP}$ & 0.012 & 0.053 & 0.103 & 0.112 & 0.305 & 0.449 & 0.099 & 0.294 & 0.443 \\
\hline & & Cauchy & 0.009 & 0.051 & 0.101 & 0.508 & 0.797 & 0.898 & 0.515 & 0.792 & 0.897 \\
\hline & \multirow{3}{*}{50} & IPS & 0.013 & 0.065 & 0.123 & 0.565 & 0.829 & 0.914 & 0.505 & 0.787 & 0.892 \\
\hline & & $\mathrm{CP}$ & 0.013 & 0.058 & 0.114 & 0.710 & 0.901 & 0.955 & 0.664 & 0.885 & 0.948 \\
\hline & & Cauchy & 0.011 & 0.053 & 0.104 & 0.999 & 1.000 & 1.000 & 0.999 & 1.000 & 1.000 \\
\hline & \multirow[t]{3}{*}{100} & IPS & 0.016 & 0.071 & 0.133 & 0.939 & 0.991 & 0.997 & 0.901 & 0.984 & 0.995 \\
\hline & & $\mathrm{CP}$ & 0.012 & 0.058 & 0.113 & 0.951 & 0.992 & 0.997 & 0.941 & 0.990 & 0.996 \\
\hline & & Cauchy & 0.010 & 0.048 & 0.094 & 1.000 & 1.000 & 1.000 & 1.000 & 1.000 & 1.000 \\
\hline & \multirow[t]{3}{*}{200} & IPS & 0.017 & 0.076 & 0.147 & 1.000 & 1.000 & 1.000 & 0.999 & 1.000 & 1.000 \\
\hline & & $\mathrm{CP}$ & 0.013 & 0.065 & 0.122 & 1.000 & 1.000 & 1.000 & 1.000 & 1.000 & 1.000 \\
\hline & & Cauchy & 0.010 & 0.048 & 0.101 & 1.000 & 1.000 & 1.000 & 1.000 & 1.000 & 1.000 \\
\hline
\end{tabular}


Table 2: Average $t$-Tests: Dependent Case

\begin{tabular}{|c|c|c|c|c|c|c|c|c|c|c|c|}
\hline \multicolumn{3}{|c|}{ Constant only } & \multicolumn{3}{|c|}{ Sizes } & \multicolumn{3}{|c|}{ Rejection Probabilities } & \multicolumn{3}{|c|}{ Size-Adjusted Powers } \\
\hline$T$ & $N$ & Tests & $1 \%$ & $5 \%$ & $10 \%$ & $1 \%$ & $5 \%$ & $10 \%$ & $1 \%$ & $5 \%$ & $10 \%$ \\
\hline \multirow[t]{12}{*}{25} & \multirow[t]{3}{*}{10} & PS & 0.012 & 0.052 & 0.102 & 0.007 & 0.037 & 0.073 & 0.006 & 0.035 & 0.072 \\
\hline & & $\mathrm{CP}$ & 0.008 & 0.046 & 0.096 & 0.019 & 0.083 & 0.155 & 0.023 & 0.090 & 0.160 \\
\hline & & Cauchy & 0.010 & 0.049 & 0.099 & 0.038 & 0.137 & 0.235 & 0.036 & 0.141 & 0.237 \\
\hline & \multirow[t]{3}{*}{50} & PS & 0.013 & 0.055 & 0.106 & 0.020 & 0.061 & 0.112 & 0.017 & 0.056 & 0.106 \\
\hline & & $\mathrm{CP}$ & 0.012 & 0.057 & 0.109 & 0.046 & 0.160 & 0.267 & 0.043 & 0.146 & 0.250 \\
\hline & & Cauchy & 0.012 & 0.051 & 0.098 & 0.117 & 0.285 & 0.396 & 0.105 & 0.281 & 0.398 \\
\hline & \multirow[t]{3}{*}{100} & PS & 0.012 & 0.055 & 0.102 & 0.020 & 0.056 & 0.092 & 0.018 & 0.052 & 0.090 \\
\hline & & $\mathrm{CP}$ & 0.016 & 0.064 & 0.117 & 0.106 & 0.276 & 0.400 & 0.079 & 0.237 & 0.365 \\
\hline & & Cauchy & 0.010 & 0.048 & 0.093 & 0.261 & 0.472 & 0.591 & 0.260 & 0.481 & 0.606 \\
\hline & \multirow[t]{3}{*}{200} & PS & 0.017 & 0.055 & 0.105 & 0.030 & 0.064 & 0.097 & 0.022 & 0.059 & 0.094 \\
\hline & & $\mathrm{CP}$ & 0.017 & 0.064 & 0.120 & 0.210 & 0.415 & 0.544 & 0.156 & 0.374 & 0.503 \\
\hline & & Cauchy & 0.013 & 0.049 & 0.091 & 0.491 & 0.672 & 0.748 & 0.453 & 0.673 & 0.758 \\
\hline \multirow[t]{12}{*}{50} & \multirow[t]{3}{*}{10} & PS & 0.011 & 0.053 & 0.103 & 0.015 & 0.059 & 0.110 & 0.013 & 0.056 & 0.108 \\
\hline & & $\mathrm{CP}$ & 0.008 & 0.043 & 0.093 & 0.022 & 0.096 & 0.175 & 0.027 & 0.108 & 0.188 \\
\hline & & Cauchy & 0.007 & 0.043 & 0.088 & 0.040 & 0.149 & 0.256 & 0.050 & 0.162 & 0.279 \\
\hline & \multirow[t]{3}{*}{50} & PS & 0.013 & 0.058 & 0.110 & 0.033 & 0.086 & 0.134 & 0.027 & 0.079 & 0.125 \\
\hline & & $\mathrm{CP}$ & 0.010 & 0.054 & 0.106 & 0.102 & 0.271 & 0.388 & 0.100 & 0.254 & 0.376 \\
\hline & & Cauchy & 0.010 & 0.050 & 0.094 & 0.277 & 0.493 & 0.596 & 0.272 & 0.494 & 0.605 \\
\hline & \multirow[t]{3}{*}{100} & PS & 0.014 & 0.059 & 0.115 & 0.075 & 0.137 & 0.190 & 0.067 & 0.129 & 0.177 \\
\hline & & $\mathrm{CP}$ & 0.012 & 0.058 & 0.112 & 0.236 & 0.437 & 0.547 & 0.216 & 0.416 & 0.530 \\
\hline & & Cauchy & 0.010 & 0.050 & 0.094 & 0.519 & 0.654 & 0.717 & 0.517 & 0.654 & 0.723 \\
\hline & \multirow[t]{3}{*}{200} & PS & 0.018 & 0.075 & 0.133 & 0.113 & 0.171 & 0.211 & 0.095 & 0.151 & 0.189 \\
\hline & & $\mathrm{CP}$ & 0.013 & 0.061 & 0.117 & 0.488 & 0.641 & 0.703 & 0.467 & 0.622 & 0.687 \\
\hline & & Cauchy & 0.010 & 0.044 & 0.087 & 0.703 & 0.763 & 0.792 & 0.703 & 0.768 & 0.799 \\
\hline \multirow[t]{12}{*}{100} & \multirow[t]{3}{*}{10} & $\mathrm{PS}$ & 0.010 & 0.052 & 0.108 & 0.052 & 0.121 & 0.180 & 0.051 & 0.118 & 0.171 \\
\hline & & $\mathrm{CP}$ & 0.008 & 0.041 & 0.088 & 0.034 & 0.119 & 0.201 & 0.039 & 0.136 & 0.218 \\
\hline & & Cauchy & 0.007 & 0.043 & 0.089 & 0.089 & 0.234 & 0.339 & 0.106 & 0.255 & 0.357 \\
\hline & \multirow[t]{3}{*}{50} & PS & 0.014 & 0.061 & 0.117 & 0.069 & 0.128 & 0.176 & 0.059 & 0.115 & 0.165 \\
\hline & & $\mathrm{CP}$ & 0.011 & 0.055 & 0.106 & 0.370 & 0.553 & 0.632 & 0.367 & 0.541 & 0.624 \\
\hline & & Cauchy & 0.010 & 0.046 & 0.094 & 0.594 & 0.705 & 0.753 & 0.596 & 0.711 & 0.755 \\
\hline & \multirow[t]{3}{*}{100} & PS & 0.014 & 0.063 & 0.122 & 0.127 & 0.193 & 0.237 & 0.120 & 0.182 & 0.224 \\
\hline & & $\mathrm{CP}$ & 0.012 & 0.058 & 0.109 & 0.538 & 0.647 & 0.695 & 0.528 & 0.636 & 0.688 \\
\hline & & Cauchy & 0.010 & 0.049 & 0.097 & 0.695 & 0.752 & 0.778 & 0.696 & 0.752 & 0.779 \\
\hline & \multirow[t]{3}{*}{200} & PS & 0.023 & 0.081 & 0.142 & 0.081 & 0.122 & 0.148 & 0.066 & 0.104 & 0.130 \\
\hline & & $\mathrm{CP}$ & 0.014 & 0.063 & 0.117 & 0.799 & 0.847 & 0.867 & 0.788 & 0.840 & 0.862 \\
\hline & & Cauchy & 0.010 & 0.048 & 0.096 & 0.873 & 0.896 & 0.909 & 0.873 & 0.897 & 0.910 \\
\hline
\end{tabular}


Table 3: Maximum Tests Along the New Contour: Independent Case

\begin{tabular}{|c|c|c|c|c|c|c|c|c|c|c|c|}
\hline \multicolumn{3}{|c|}{ Constant only } & \multicolumn{3}{|c|}{ Sizes } & \multicolumn{3}{|c|}{ Rejection Probabilities } & \multicolumn{3}{|c|}{ Size-Adjusted Powers } \\
\hline$T$ & $N$ & Tests & $1 \%$ & $5 \%$ & $10 \%$ & $1 \%$ & $5 \%$ & $10 \%$ & $1 \%$ & $5 \%$ & $10 \%$ \\
\hline \multirow[t]{8}{*}{25} & \multirow[t]{2}{*}{10} & $\mathrm{CP}$ & 0.011 & 0.051 & 0.108 & 0.024 & 0.103 & 0.187 & 0.020 & 0.099 & 0.179 \\
\hline & & Cauchy & 0.008 & 0.037 & 0.076 & 0.049 & 0.169 & 0.272 & 0.059 & 0.209 & 0.318 \\
\hline & \multirow[t]{2}{*}{50} & $\mathrm{CP}$ & 0.012 & 0.057 & 0.119 & 0.038 & 0.136 & 0.225 & 0.035 & 0.124 & 0.201 \\
\hline & & Cauchy & 0.005 & 0.029 & 0.061 & 0.066 & 0.184 & 0.278 & 0.104 & 0.247 & 0.371 \\
\hline & \multirow[t]{2}{*}{100} & $\mathrm{CP}$ & 0.013 & 0.061 & 0.122 & 0.057 & 0.172 & 0.270 & 0.048 & 0.148 & 0.238 \\
\hline & & Cauchy & 0.003 & 0.023 & 0.052 & 0.083 & 0.213 & 0.314 & 0.137 & 0.306 & 0.419 \\
\hline & \multirow[t]{2}{*}{200} & $\mathrm{CP}$ & 0.016 & 0.074 & 0.136 & 0.072 & 0.200 & 0.305 & 0.052 & 0.150 & 0.245 \\
\hline & & Cauchy & 0.003 & 0.017 & 0.044 & 0.098 & 0.237 & 0.336 & 0.198 & 0.351 & 0.453 \\
\hline \multirow[t]{8}{*}{50} & \multirow[t]{2}{*}{10} & $\mathrm{CP}$ & 0.009 & 0.050 & 0.102 & 0.033 & 0.126 & 0.227 & 0.036 & 0.126 & 0.224 \\
\hline & & Cauchy & 0.009 & 0.043 & 0.088 & 0.085 & 0.258 & 0.385 & 0.088 & 0.277 & 0.414 \\
\hline & \multirow[t]{2}{*}{50} & $\mathrm{CP}$ & 0.010 & 0.054 & 0.103 & 0.072 & 0.207 & 0.318 & 0.068 & 0.196 & 0.313 \\
\hline & & Cauchy & 0.007 & 0.038 & 0.077 & 0.199 & 0.387 & 0.503 & 0.232 & 0.428 & 0.551 \\
\hline & \multirow[t]{2}{*}{100} & $\mathrm{CP}$ & 0.016 & 0.064 & 0.122 & 0.114 & 0.271 & 0.388 & 0.089 & 0.235 & 0.348 \\
\hline & & Cauchy & 0.006 & 0.031 & 0.069 & 0.284 & 0.469 & 0.569 & 0.340 & 0.525 & 0.620 \\
\hline & \multirow[t]{2}{*}{200} & $\mathrm{CP}$ & 0.012 & 0.060 & 0.110 & 0.157 & 0.331 & 0.450 & 0.139 & 0.304 & 0.428 \\
\hline & & Cauchy & 0.006 & 0.029 & 0.061 & 0.357 & 0.529 & 0.626 & 0.423 & 0.596 & 0.694 \\
\hline \multirow[t]{8}{*}{100} & \multirow[t]{2}{*}{10} & $\mathrm{CP}$ & 0.008 & 0.047 & 0.095 & 0.070 & 0.203 & 0.314 & 0.081 & 0.210 & 0.322 \\
\hline & & Cauchy & 0.008 & 0.044 & 0.092 & 0.255 & 0.453 & 0.559 & 0.268 & 0.471 & 0.574 \\
\hline & \multirow[t]{2}{*}{50} & $\mathrm{CP}$ & 0.011 & 0.053 & 0.105 & 0.240 & 0.438 & 0.561 & 0.234 & 0.427 & 0.555 \\
\hline & & Cauchy & 0.008 & 0.044 & 0.086 & 0.623 & 0.770 & 0.832 & 0.634 & 0.785 & 0.844 \\
\hline & \multirow[t]{2}{*}{100} & $\mathrm{CP}$ & 0.012 & 0.054 & 0.102 & 0.267 & 0.468 & 0.579 & 0.252 & 0.457 & 0.575 \\
\hline & & Cauchy & 0.007 & 0.043 & 0.088 & 0.621 & 0.758 & 0.815 & 0.646 & 0.771 & 0.825 \\
\hline & \multirow[t]{2}{*}{200} & $\mathrm{CP}$ & 0.009 & 0.044 & 0.086 & 0.406 & 0.588 & 0.683 & 0.413 & 0.603 & 0.705 \\
\hline & & Cauchy & 0.008 & 0.040 & 0.080 & 0.780 & 0.867 & 0.902 & 0.793 & 0.879 & 0.914 \\
\hline \multirow[t]{8}{*}{200} & \multirow[t]{2}{*}{10} & $\mathrm{CP}$ & 0.009 & 0.048 & 0.098 & 0.319 & 0.547 & 0.662 & 0.331 & 0.553 & 0.667 \\
\hline & & Cauchy & 0.009 & 0.044 & 0.088 & 0.528 & 0.743 & 0.828 & 0.548 & 0.756 & 0.842 \\
\hline & \multirow[t]{2}{*}{50} & $\mathrm{CP}$ & 0.008 & 0.042 & 0.086 & 0.501 & 0.664 & 0.743 & 0.525 & 0.684 & 0.762 \\
\hline & & Cauchy & 0.009 & 0.050 & 0.095 & 0.745 & 0.854 & 0.899 & 0.756 & 0.854 & 0.901 \\
\hline & \multirow[t]{2}{*}{100} & $\mathrm{CP}$ & 0.006 & 0.041 & 0.077 & 0.551 & 0.690 & 0.758 & 0.583 & 0.714 & 0.780 \\
\hline & & Cauchy & 0.009 & 0.047 & 0.091 & 0.748 & 0.840 & 0.876 & 0.753 & 0.844 & 0.881 \\
\hline & \multirow[t]{2}{*}{200} & $\mathrm{CP}$ & 0.006 & 0.030 & 0.068 & 0.646 & 0.764 & 0.820 & 0.688 & 0.797 & 0.846 \\
\hline & & Cauchy & 0.007 & 0.042 & 0.085 & 0.857 & 0.912 & 0.935 & 0.866 & 0.919 & 0.940 \\
\hline
\end{tabular}


Table 4: Maximum Tests Along the New Contour: Dependent Case

\begin{tabular}{|c|c|c|c|c|c|c|c|c|c|c|c|}
\hline \multicolumn{3}{|c|}{ Constant only } & \multicolumn{3}{|c|}{ Sizes } & \multicolumn{3}{|c|}{ Rejection Probabilities } & \multicolumn{3}{|c|}{ Size-Adjusted Powers } \\
\hline$T$ & $N$ & Tests & $1 \%$ & $5 \%$ & $10 \%$ & $1 \%$ & $5 \%$ & $10 \%$ & $1 \%$ & $5 \%$ & $10 \%$ \\
\hline \multirow[t]{8}{*}{25} & \multirow[t]{2}{*}{10} & $\mathrm{CP}$ & 0.015 & 0.065 & 0.123 & 0.026 & 0.104 & 0.186 & 0.016 & 0.080 & 0.153 \\
\hline & & Cauchy & 0.013 & 0.055 & 0.100 & 0.050 & 0.151 & 0.247 & 0.036 & 0.141 & 0.248 \\
\hline & \multirow[t]{2}{*}{50} & $\mathrm{CP}$ & 0.012 & 0.059 & 0.119 & 0.031 & 0.106 & 0.184 & 0.026 & 0.092 & 0.159 \\
\hline & & Cauchy & 0.005 & 0.027 & 0.056 & 0.042 & 0.117 & 0.190 & 0.063 & 0.174 & 0.270 \\
\hline & \multirow[t]{2}{*}{100} & $\mathrm{CP}$ & 0.014 & 0.061 & 0.119 & 0.040 & 0.135 & 0.218 & 0.031 & 0.120 & 0.190 \\
\hline & & Cauchy & 0.003 & 0.022 & 0.048 & 0.051 & 0.134 & 0.211 & 0.096 & 0.214 & 0.309 \\
\hline & \multirow[t]{2}{*}{200} & $\mathrm{CP}$ & 0.015 & 0.063 & 0.120 & 0.049 & 0.144 & 0.232 & 0.033 & 0.118 & 0.207 \\
\hline & & Cauchy & 0.003 & 0.018 & 0.042 & 0.052 & 0.144 & 0.228 & 0.107 & 0.251 & 0.344 \\
\hline \multirow[t]{8}{*}{50} & \multirow[t]{2}{*}{10} & $\mathrm{CP}$ & 0.015 & 0.073 & 0.131 & 0.035 & 0.128 & 0.219 & 0.026 & 0.092 & 0.169 \\
\hline & & Cauchy & 0.014 & 0.059 & 0.108 & 0.070 & 0.200 & 0.296 & 0.056 & 0.179 & 0.286 \\
\hline & \multirow[t]{2}{*}{50} & $\mathrm{CP}$ & 0.014 & 0.057 & 0.112 & 0.055 & 0.166 & 0.263 & 0.043 & 0.149 & 0.246 \\
\hline & & Cauchy & 0.006 & 0.036 & 0.076 & 0.139 & 0.285 & 0.382 & 0.157 & 0.327 & 0.425 \\
\hline & \multirow[t]{2}{*}{100} & $\mathrm{CP}$ & 0.013 & 0.058 & 0.114 & 0.077 & 0.188 & 0.280 & 0.065 & 0.173 & 0.259 \\
\hline & & Cauchy & 0.005 & 0.031 & 0.067 & 0.190 & 0.335 & 0.424 & 0.232 & 0.386 & 0.483 \\
\hline & \multirow[t]{2}{*}{200} & $\mathrm{CP}$ & 0.013 & 0.059 & 0.110 & 0.110 & 0.239 & 0.330 & 0.097 & 0.224 & 0.312 \\
\hline & & Cauchy & 0.005 & 0.026 & 0.058 & 0.274 & 0.415 & 0.496 & 0.323 & 0.479 & 0.556 \\
\hline \multirow[t]{8}{*}{100} & \multirow[t]{2}{*}{10} & $\mathrm{CP}$ & 0.016 & 0.072 & 0.132 & 0.050 & 0.145 & 0.217 & 0.039 & 0.113 & 0.183 \\
\hline & & Cauchy & 0.014 & 0.061 & 0.118 & 0.137 & 0.274 & 0.357 & 0.110 & 0.251 & 0.334 \\
\hline & \multirow[t]{2}{*}{50} & $\mathrm{CP}$ & 0.013 & 0.057 & 0.104 & 0.154 & 0.304 & 0.391 & 0.140 & 0.291 & 0.382 \\
\hline & & Cauchy & 0.010 & 0.043 & 0.086 & 0.439 & 0.573 & 0.641 & 0.440 & 0.586 & 0.655 \\
\hline & \multirow[t]{2}{*}{100} & $\mathrm{CP}$ & 0.011 & 0.048 & 0.095 & 0.181 & 0.314 & 0.399 & 0.174 & 0.317 & 0.406 \\
\hline & & Cauchy & 0.007 & 0.039 & 0.081 & 0.485 & 0.588 & 0.646 & 0.505 & 0.606 & 0.661 \\
\hline & \multirow[t]{2}{*}{200} & $\mathrm{CP}$ & 0.009 & 0.041 & 0.082 & 0.299 & 0.452 & 0.534 & 0.304 & 0.474 & 0.559 \\
\hline & & Cauchy & 0.008 & 0.042 & 0.079 & 0.674 & 0.753 & 0.792 & 0.683 & 0.765 & 0.806 \\
\hline \multirow[t]{8}{*}{200} & \multirow[t]{2}{*}{10} & $\mathrm{CP}$ & 0.015 & 0.068 & 0.124 & 0.166 & 0.309 & 0.397 & 0.145 & 0.273 & 0.363 \\
\hline & & Cauchy & 0.016 & 0.063 & 0.118 & 0.318 & 0.519 & 0.620 & 0.273 & 0.480 & 0.591 \\
\hline & \multirow[t]{2}{*}{50} & $\mathrm{CP}$ & 0.010 & 0.049 & 0.093 & 0.225 & 0.336 & 0.399 & 0.224 & 0.338 & 0.407 \\
\hline & & Cauchy & 0.011 & 0.048 & 0.092 & 0.572 & 0.673 & 0.720 & 0.565 & 0.676 & 0.726 \\
\hline & \multirow[t]{2}{*}{100} & $\mathrm{CP}$ & 0.006 & 0.035 & 0.072 & 0.282 & 0.379 & 0.436 & 0.307 & 0.401 & 0.467 \\
\hline & & Cauchy & 0.009 & 0.045 & 0.090 & 0.618 & 0.693 & 0.730 & 0.623 & 0.699 & 0.734 \\
\hline & \multirow[t]{2}{*}{200} & $\mathrm{CP}$ & 0.006 & 0.031 & 0.063 & 0.370 & 0.459 & 0.514 & 0.390 & 0.496 & 0.553 \\
\hline & & Cauchy & 0.009 & 0.047 & 0.090 & 0.754 & 0.802 & 0.830 & 0.755 & 0.806 & 0.834 \\
\hline
\end{tabular}

